\title{
Mobile and Wearable Sensing Frameworks for mHealth Studies and Applications A Systematic Review
}

\author{
Kumar, Devender; Jeuris, Steven; Bardram, Jakob E.; Dragoni, Nicola
}

\section{Published in:}

Acm Transactions on Computing and Healthcare

Link to article, DOI:

$10.1145 / 3422158$

Publication date:

2021

Document Version

Publisher's PDF, also known as Version of record

Link back to DTU Orbit

Citation (APA):

Kumar, D., Jeuris, S., Bardram, J. E., \& Dragoni, N. (2021). Mobile and Wearable Sensing Frameworks for mHealth Studies and Applications: A Systematic Review. Acm Transactions on Computing and Healthcare, 2(1), [8]. https://doi.org/10.1145/3422158

\section{General rights}

Copyright and moral rights for the publications made accessible in the public portal are retained by the authors and/or other copyright owners and it is a condition of accessing publications that users recognise and abide by the legal requirements associated with these rights.

- Users may download and print one copy of any publication from the public portal for the purpose of private study or research.

- You may not further distribute the material or use it for any profit-making activity or commercial gain

- You may freely distribute the URL identifying the publication in the public portal 


\title{
Mobile and Wearable Sensing Frameworks for mHealth Studies and Applications: A Systematic Review
}

\author{
DEVENDER KUMAR, STEVEN JEURIS, JAKOB E. BARDRAM, and NICOLA DRAGONI, \\ Technical University of Denmark
}

\begin{abstract}
With the widespread use of smartphones and wearable health sensors, a plethora of mobile health (mHealth) applications to track well-being, run human behavioral studies, and clinical trials have emerged in recent years. However, the design, development, and deployment of mHealth applications is challenging in many ways. To address these challenges, several generic mobile sensing frameworks have been researched in the past decade. Such frameworks assist developers and researchers in reducing the complexity, time, and cost required to build and deploy health-sensing applications. The main goal of this article is to provide the reader with an overview of the state-of-the-art of health-focused generic mobile and wearable sensing frameworks. This review gives a detailed analysis of functional and non-functional features of existing frameworks, the health studies they were used in, and the stakeholders they support. Additionally, we also analyze the historical evolution, uptake, and maintenance after the initial release. Based on this analysis, we suggest new features and opportunities for future generic mHealth sensing frameworks.
\end{abstract}

CCS Concepts: • Human-centered computing $\rightarrow$ Ubiquitous and mobile computing; • Software and its engineering $\rightarrow$ Development frameworks and environments; • Applied computing $\rightarrow$ Health informatics;

Additional Key Words and Phrases: mHealth sensing, mobile sensing, wearable sensing, mHealth frameworks, mobile sensing frameworks

ACM Reference format:

Devender Kumar, Steven Jeuris, Jakob E. Bardram, and Nicola Dragoni. 2020. Mobile and Wearable Sensing Frameworks for mHealth Studies and Applications: A Systematic Review. ACM Trans. Comput. Healthcare 2, 1, Article 8 (December 2020 ), 28 pages.

https://doi.org/10.1145/3422158

\footnotetext{
This work is partly funded by the Copenhagen Center for Health Technology and the Innovation Foundation of Denmark under grants 6153-00009B and 6152-00005B for projects: REAFEL: Reaching the Frail Elderly Patient for Optimizing Diagnosis of Atrial Fibrillation (http: //www.cachet.dk/research/research_projects/REAFEL) and BHRP: Biometric Healthcare Research Platform for Research in Psychiatric and Neurological Diseases using Sensor Technologies (http://www.cachet.dk/research/research_projects/bhrp).

Authors' addresses: D. Kumar, S. Jeuris, and J. E. Bardram, Department of Health Technology, Technical University of Denmark, Richard Pedersens Plads, Building 324, Kgs. Lyngby, DK-2800; emails: \{deku, sjeu, jakba\}@dtu.dk; N. Dragoni, Dept. of Applied Mathematics \& Computer Science, Technical University of Denmark, Richard Pedersens Plads, Building 322, Kgs. Lyngby, DK-2800; email: ndra@dtu.dk.

Permission to make digital or hard copies of part or all of this work for personal or classroom use is granted without fee provided that copies are not made or distributed for profit or commercial advantage and that copies bear this notice and the full citation on the first page. Copyrights for third-party components of this work must be honored. For all other uses, contact the owner/author(s).

(c) 2020 Copyright held by the owner/author(s).

2637-8051/2020/12-ART8

https://doi.org/10.1145/3422158
}

ACM Transactions on Computing for Healthcare, Vol. 2, No. 1, Article 8. Publication date: December 2020. 


\section{INTRODUCTION}

In the past decade, rapid improvements in processing power, network speed, storage, and the addition of integrated sensors on mobile phones, combined with wearable sensors, have paved the way for new opportunities in research fields such as mobile crowdsensing (MCS), ubiquitous computing, and pervasive health. Today most smartphones are equipped with a wide range of sensing capabilities, such as motion and direction (accelerometer and gyroscope), position (GPS), light intensity, atmospheric pressure (barometer), temperature, proximity, and connectivity (Bluetooth and Wi-Fi). In addition, wearable devices such as activity trackers and medical devices can be connected to the smartphone, which then works as the hub for data collection and processing. Due to the widespread availability of smartphones (nearly 2.5B globally [46]), researchers use them for in situ data collection for social, environmental, and mobile health (mHealth) studies. Among others, mHealth in particular has shown growing scientific and commercial interest and has been used for next-generation health research, including in situ monitoring and just-in-time (JIT) interventions [2, 10]. Over the years, several mHealth based studies have been conducted in areas such as heart arrhythmia detection, cardiac rehabilitation training, stress, depression, and behavior change $[14,36,67,87]$. However, to conduct these mHealth studies, researchers may need to implement their own study specific sensing, data storage, and data analysis from scratch, as well as handle nonfunctional technical challenges such as smartphone resource optimization, configuration of sensors, support for longitudinal data storage and processing, and constant upgrading of the low-level sensing integration to different operating systems (e.g., iOS vs. Android) and smartphone hardware models. All of this requires substantial technical skills and many resources, which is costly and time-consuming. To address these challenges and to support the development and deployment of mHealth studies, researchers have been designing and building more generic and reusable mHealth sensing frameworks. These frameworks provide configurable libraries, modules, and plugins for mobile and wearable sensing, data storage, management, and analysis. Over the years, generic sensing frameworks such as Funf [3], AWARE [24], mCerebrum [35], Beiwe [85], and RADAR-base [68] have been designed and implemented. Such frameworks have typically been released as open source for other researchers to download, adapt, and (re)use. The overall purpose of these frameworks is to help researchers to easily design and deploy mHealth studies with no-or very limited-need for actually programming the sensing technology.

This article presents a systematic literature review of existing mobile and wearable sensing frameworks for mHealth. Such a review is useful for clinical researchers who want to engage in mHealth research studies and are looking for a sensing framework to support their research, as well as for technical researchers interested in existing frameworks to use, extend, or adapt in the design of their own technical solutions and applications. Prior reviews of sensing frameworks have focused on more technical issues such as privacy, resource management strategies, and energy efficiency. For instance, Wang et al. [86] presented a review of various state-of-the-art energy saving techniques in mobile crowdsensing (MCS) applications. Similarly, Christin et al. [16] did a survey on privacy in participatory mobile sensing applications. The review by Khan et al. [42] provided an overview of the state-of-the-art of mobile phone sensing applications in several domains such as traffic and environmental monitoring. Hence, to the best of our knowledge, this systematic review is the first to focus on mHealth sensing frameworks. The review focuses on generic sensing frameworks for mHealth, which is distinct from use-casespecific sensing applications. Generic sensing frameworks can typically be configured to support several different types of studies (i.e., use-cases/diseases) or can be used to build new sensing applications, whereas use-casespecific sensing applications are tied to a particular study.

This review is conducted as a systematic literature review (SLR) describing the state-of-the-art of currently available generic frameworks that can be used for creating and implementing mHealth studies and applications. The review focuses on describing the functional and non-functional features, historic evolution, maintenance, uptake, and supported stakeholders of the different frameworks. Based on this overview, the article highlights and discusses open issues and potential new features that could be relevant to implement in the further development of such frameworks. The contribution of this systematic literature review (SLR) can be summarized as follows: 
- It identifies, summarizes, and analyzes existing generic mHealth sensing frameworks and presents the health studies, application areas, and stakeholders each of them support.

- It provides an overview of common functional and non-functional features in the identified frameworks.

- It examines the historical evolution and the uptake of these mHealth sensing frameworks, including how well they are maintained and updated after their initial release.

- Based on this analysis, the review identifies and discusses open issues in contemporary research on mHealth sensing frameworks.

The rest of the article is organized as follows: Section 2 describes the systematic review methods, including the research questions, search and screening strategy, inclusion and exclusion criteria, and a description of the data extraction procedure. Section 3 through Section 6 present a detailed analysis of all the functional and nonfunctional features, health areas and applications, and historical evolution of generic mHealth sensing frameworks. Section 7 provides a discussion of the results, including trends in the research of generic sensing frameworks, their implications for healthcare, as well as new potential features needed in the further development of such sensing frameworks, and potential threats to the validity of this review. Section 8 concludes the article.

\section{SYSTEMATIC REVIEW PROCEDURE}

In this SLR, we applied the review strategy for conducting SLR in software engineering as proposed by Kitchenham and Charters [43] with a slight modification in search strategy to make it a better fit for this review in the field of mHealth. Specifically, in addition to the proposed search strategy, the backward snowballing technique [90] was applied for finding additional relevant papers. This implies iteratively screening the list of references of the already-included papers in the review.

\subsection{Identifying the Need for the Review}

Within the past decade, there has been a growing interest in using mobile and wearable technology for collecting contextual, behavioral, and health-related data "in-the-wild," i.e., data that are not acquired in a clinical and/or laboratory setting, but are collected continuously from users in their everyday life. For this purpose, a range of generic mobile-wearable data sampling platforms and frameworks have been designed and released for general use. To search for a systematic overview of existing framework for such mobile and wearable sensing for mHealth purposes, we conducted searches for "systematic literature reviews" in online databases such as ACM, IEEE, Google Scholar, and Scopus using terms such as "mobile/smartphone sensing framework," "crowdsensing frameworks," "sensing framework," and "mobile data collection" in combination with "health" or "behavior sensing" to find any existing SLRs summarizing different generic mobile sensing frameworks for mHealth applications. The search indicated that no overview of this research exists. Therefore, an SLR to summarize and discuss the current state-of-the-art in health-focused generic mobile sensing frameworks is relevant and needed.

\subsection{Research Questions}

The objective of this review is to identify and provide an overview of all relevant mobile/wearable sensing platforms and frameworks for mHealth and provide an overview of their features (functional and non-functional), which health domain they are used in, and how they have evolved historically and are maintained over time. This overall objective leads to the following four research questions (RQs):

RQ1: Which health-focused generic mobile and wearable sensing platforms and frameworks exist, and which health studies, application areas, and stakeholder do they target?

RQ2: What functional features are supported by these frameworks?

RQ3: What are the non-functional features of these frameworks in terms of extensibility, scalability, security, privacy, license model, and documentation? 
Table 1. Number of Papers per Database

\begin{tabular}{|l|c|}
\hline Database & Search results \\
\hline IEEE & 330 \\
ACM & 226 \\
Scopus & 1,058 \\
\hline
\end{tabular}

RQ4: How have these frameworks evolved over time, and how are they reused and maintained after their initial release?

\subsection{Search Strategy}

The first step of the search strategy was to identify a search string. The search string was constructed by following the guidelines from Khakurel et al. [41] and involved four components. First, we selected keywords used in our previously defined RQs. In addition, we used initial Google Scholar search results to check which keywords are used in popular framework papers. Second, we identified synonyms, acronyms, and alternative phrasings for these keywords. For example, "mobile sensing" for "smartphone sensing," "mobile crowdsensing" for "smartphone crowd sensing," and "behaviour" for "behavior." Third, we merged all synonyms, acronyms, and alternative phrasings using "OR" operations. Finally, all the terms were combined to construct the final search string:

("mobile sensing" OR "smartphone sensing" OR "context sensing" OR "wearable sensing" OR "mobile platform" OR "smartphone platform" OR "mobile context" OR "smartphone context" OR "mobile data collection" OR "sensing framework" OR "data collection platform" OR "smartphone data collection" OR "mobile crowdsensing" OR "smartphone crowdsensing" OR "mobile crowd sensing" OR "smartphone crowd sensing")

AND ("health" OR "human behaviour" OR "human behavior").

In the second step, the first author of this article (DK) used the finalized search string on Dec 20, 2018, to search three electronic databases: (1) IEEE Xplore, (2) ACM Digital Library, and (3) Scopus, limiting the search to publications between 2008-2018 inclusive. The database search was performed on title, abstract, and keywords. These databases were chosen because of their relevance to the field of mobile and wearable technology. Table 1 lists the number of search results for each database.

\subsection{Inclusion and Exclusion Criteria}

The aim of applying inclusion criterias (ICs) and exclusion criterias (ECs) is to extract only publications relevant to the objective of this SLR. We used the following set of inclusion criterias (ICs) and exclusion criterias (ECs):

IC1: Papers should describe a framework that is generic and supports designing and/or building new sensing applications on top of it, rather than being a use-case or application-specific system.

IC2: Papers should include a reasonably detailed description of the sensing framework's architecture and technical implementation.

IC3: Papers should focus on potential use cases in health, wellness, or behavior sensing.

IC4: Publication date should be on or after the year 2008 (the iPhone was introduced late 2007).

EC1: Papers should not describe a system that is specific to one study or that is not generic enough to support building other mHealth applications than the one described.

EC2: Papers that do not focus on health or well-being. 


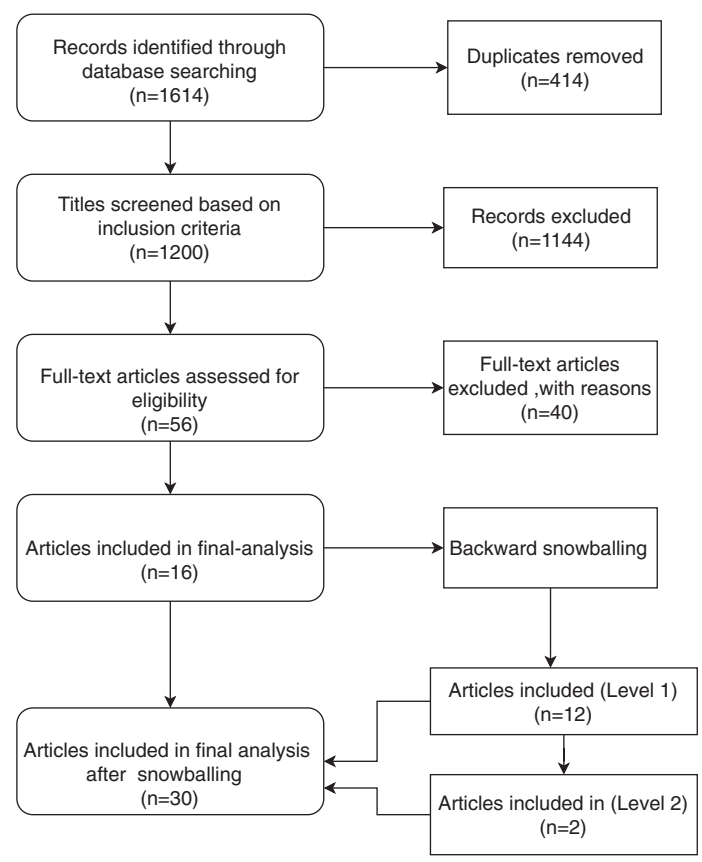

Fig. 1. Review flowchart for selection of papers.

\subsection{Screening Process}

Figure 1 illustrates the different phases and the number of retained papers in each phase of the screening process. The initial search found 1,614 papers, from which 414 duplicates were removed. The remaining 1,200 papers were then screened based on their titles, adhering to the above-listed ICs and ECs, which removed 1,144 irrelevant papers. Most of these papers were removed due to EC1, since the title often revealed whether a paper presented a use-case-specific sensing application or a generic framework. This resulted in a total of 56 papers for full-text screening, out of which 16 papers were retained for further analysis.

Next, backward snowballing [90] was applied. This implies iteratively looking at the list of references of the included papers and screening them to find new potential papers to include. Through backward snowballing, we identified several papers that used alternative phrasings of "data collection platform" or "mobile sensing frameworks," which we did not anticipate, such as "digital phenotyping" and "social psychology sensing toolkit." Therefore, these papers were only found through backward snowballing, highlighting the effectiveness of this approach. We primarily focused on the references within the "Related Work" sections of the papers. In a first iteration, we included 12 new papers by following references in the initial 16. In a second iteration, we included two new papers. These last two inclusions did not lead to any new identified papers, thereby concluding the snowballing process. Thus, in total, 14 additional papers $(12+2)$ were included as part of the snowballing process and at the end of the screening process, 30 papers were included.

\subsection{Additional Frameworks}

Many of the papers include references to open-source or commercial mobile and wearable sensing frameworks. These frameworks are not published in peer-reviewed scientific venues, but are still similar to the systems found through the systematic literature search and have been widely used for building mHealth applications. To make our review more inclusive, we will also include and discuss these unpublished frameworks. However, given that 
they do not have an associated publication, we want to emphasize that their presented data have not been obtained systematically as has been done for the frameworks with a matching publication. Instead, we extracted available information from their website and open-source code base, as available at the time of writing this review. The data tables presented in this review will clearly distinguish between scientifically published frameworks (white background) and unpublished frameworks (gray background).

\subsection{Data Extraction, Categorization, and Labeling}

The categorization and labeling schemes were created iteratively and were continuously verified through a collaborative process involving all authors. This includes the labeling of application areas, stakeholder classification, sensing typology, and the classification of functional and non-functional features. Full data extraction, labeling, and categorization of the 30 papers was initially done by the first author (DK) and then validated by the second author (SJ). We found two pairs of papers describing the same sensing framework, and one paper from each of these two pairs was removed. Thus, in total, we analyzed 28 distinct sensing frameworks, as listed in Table 2. For information not contained within the papers-such as software license, available documentation, and last update to the code base-we investigated the open-source repositories of the frameworks, if available.

\section{FRAMEWORK OVERVIEW, APPLICATIONS AREAS, AND STAKEHOLDERS}

This section outlines the results with respect to the first research question (RQ1) introduced in Section 2.2.

\subsection{Frameworks}

Table 2 provides an overview of all identified frameworks. In total, 28 frameworks have been published in scientific peer-reviewed literature, while 9 frameworks are unpublished (shown on a gray background). In the presentation and discussion of the findings, we will focus on the 28 scientifically published frameworks, unless mentioned otherwise.

We classify 9 frameworks as "end-to-end," as these frameworks provide support for all aspects of running a mHealth study, including data collection and storage, data processing, visualization, participant recruitment, and monitoring study progress. The majority of frameworks $(N=20)$ are built for Android, followed by iOS $(N=7)$ and Nokia $(N=3)$; six frameworks support both Android and iOS operating systems.

\subsection{Application Area}

Frameworks included in this review are generic and intend to support the design and implementation of mHealth applications in a broad sense. However, to provide an overview of some of the health domain(s) a framework has been used in, we looked at case studies reported in the papers documenting the framework, in papers citing the framework paper, and on the framework's website, if available. Table 3 lists examples of health studies that were implemented using the corresponding frameworks. We find that despite the intention of these frameworks to be general-purpose, most of them still have only been used in a limited number of studies. As shown in Figure 2, the mHealth studies can broadly be categorized into three overlapping categories: behavioral, mental health, and physiological health studies.

In behavioral studies, a sensing framework is used to build applications that collect behavioral data (e.g., diet, physical activity). The collected data can be used by behavioral health professionals to understand which behavior contributes to individuals' health condition. Examples include: risky behaviors and HIV transmission using AndWellness [32]; obesity monitoring using HealthOS [48]; StudentLife [87] for assessing behavioral trends, health, and academic performance of college students using Jigsaw [51]; and behavioral correlation between location, social context, and mobility context using AWARE [24]. Out of 28 frameworks, 20 have been used in studies or applications related to human behavioral studies. 


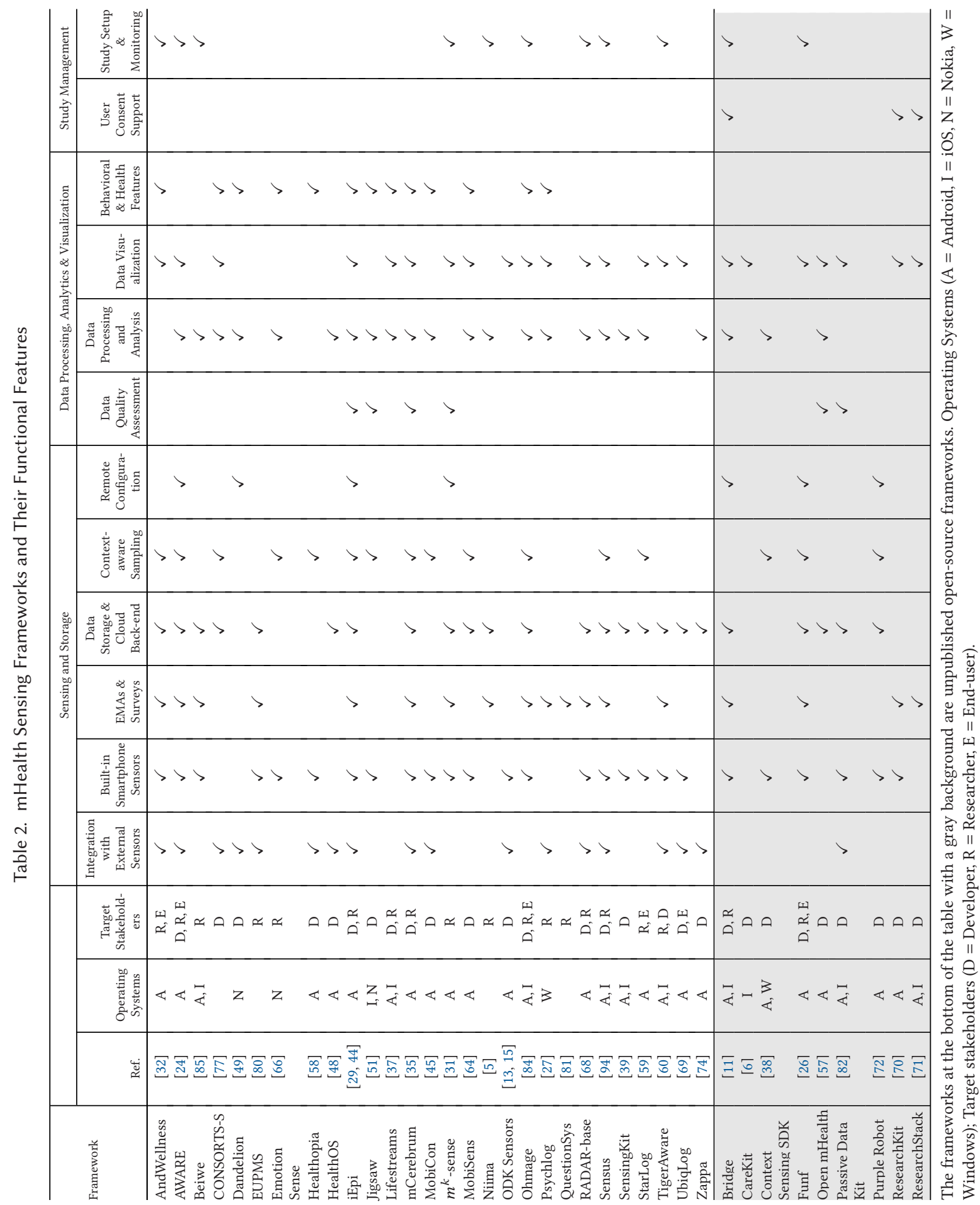


Table 3. Health Applications Implemented Using the Frameworks

\begin{tabular}{|c|c|}
\hline Framework & Health Studies \\
\hline AndWellness & $\begin{array}{l}\text { Risky behaviors and HIV transmission [32], Behaviors and emotions of young breast cancer } \\
\text { survivors [32] }\end{array}$ \\
\hline AWARE & $\begin{array}{l}\text { Alcohol use events JIT intervention [8], Symptom severity during chemotherapy [50], Detecting } \\
\text { drinking episodes [9] }\end{array}$ \\
\hline Beiwe & Schizophrenia spectrum illness [85] \\
\hline CONSORTS-S & Physiological symptom monitoring [78] \\
\hline Dandelion & Heart-rate and EKG monitoring [49], Fall detection [49] \\
\hline Emotion Sense & Social psychology [66] \\
\hline EUPMS & Remote therapeutic interventions [79] \\
\hline HealthOS & ObeCure: obesity monitoring [48] \\
\hline iEpi & $\begin{array}{l}\text { Study of health behavior in pregnant mothers diagnosed with gestational diabetes [44], Gamified } \\
\text { intervention for healthy behavior [44] }\end{array}$ \\
\hline Jigsaw & $\begin{array}{l}\text { StudentLife: assessing mental health, academic performance, and behavioral trends of college } \\
\text { students [87] }\end{array}$ \\
\hline Lifestreams & Diet, stress, and exercise in young moms [37], Family wellness study [37] \\
\hline mCerebrum & Smoking \& stress [35], Heart failure [35] \\
\hline MobiSens & Remote elderly care [91], Mental health monitoring [92] \\
\hline$m^{k}$-sense & Thought and life logging-Mental time travel [31] \\
\hline ODK Sensors & Heart rate monitoring [15], Diagnose childhood pneumonia [13] \\
\hline Ohmage & $\begin{array}{l}\text { Moms: Studying diet, stress, and exercise-related risk factors for CVD in young mothers [84], } \\
\text { PREEMPT: N-of-1 trials using mHealth in chronic pain [84] }\end{array}$ \\
\hline Psychlog & Arousal and psychological stress [27] \\
\hline QuestionSys & Remote therapeutic interventions [79] \\
\hline RADAR-base & Depression and epilepsy [83] \\
\hline Sensus & $\begin{array}{l}\text { Hourly activity sampling in behavioral activation [73], Social interaction anxiety scale } \\
\text { assessment [94] }\end{array}$ \\
\hline StarLog & Behavior analysis [59] \\
\hline TigerAware & Diabetes self-management study [60], Drink and drive [60] \\
\hline UbiqLog & Mood and sleep [69] \\
\hline Zappa & Cloud Rehab: Tracking patients with severe brain damage [74] \\
\hline Bridge & Blood pressure and stress levels tracking [54], Mole Mapper [88] \\
\hline CareKit & $\begin{array}{l}\text { A symptom tracking and reporting instrument mobile application for central nervous system } \\
\text { cancer patients [47] }\end{array}$ \\
\hline Funf & EMA of day-to-day mood [7], Modeling and discovering human behavior [52] \\
\hline Open mHealth & Post-traumatic stress disorder (PTSD) [55], Type 1 diabetes self-monitoring case study [56] \\
\hline Purple Robot & Depressive symptom severity in daily-life behavior [75] \\
\hline ResearchKit & mPower study (Parkinson’s disease) [12], Mole Mapper [88],C3-PRO [65] \\
\hline ResearchStack & C3-PRO [65] \\
\hline
\end{tabular}

Mental health studies are built to assess the psychological, emotional, social conditions of human subjects, e.g., to study bipolar disorder, schizophrenia, depression, and anxiety disorder. Although there is overlap between behavioral and mental health disorders, not all mental health disorders are a result of behavioral issues. Of the 28 frameworks, 14 have been used in applications studies related to mental health. Examples include: monitoring schizophrenia spectrum illness using Beiwe [85], and depression and epilepsy using RADAR-base [68]. 


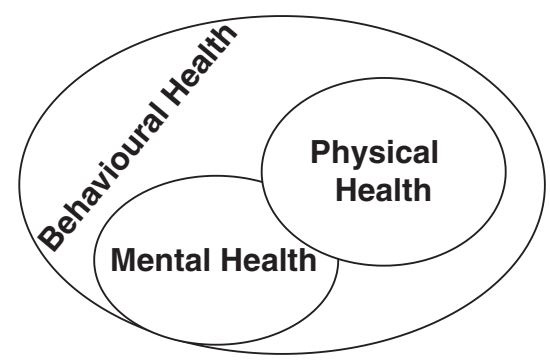

Fig. 2. Behavioral health is a superset of mental and physical health that looks at how behaviors impact health.

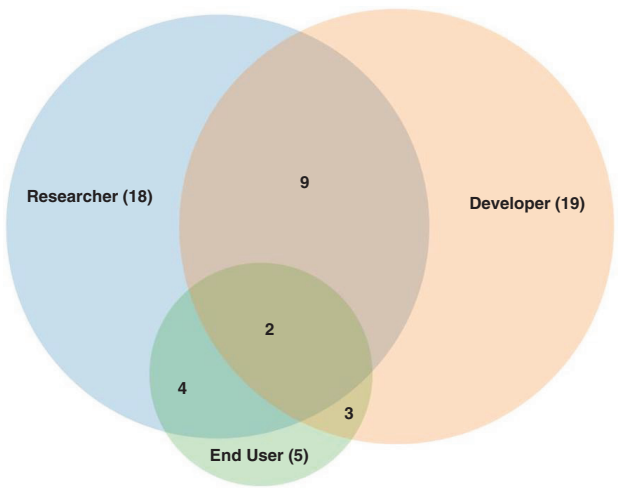

Fig. 3. Number of frameworks supporting different stakeholders.

Physiological health studies involve sensing physiological data such as heart rate, blood pressure, ECG, and skin temperature. Examples include: heart rate monitoring built using Dandelion [49] and heart failure detection using mCerebrum [35]. Of the 28 frameworks, 13 have been used in studies or applications related to physiological health sensing.

\subsection{Stakeholders}

Since the goal of these general-purpose health sensing frameworks is to support a wide range of health domains and studies with a wide range of potential features, they are also designed to target different stakeholders, depending on their focus. During the labeling of the included papers, we identified the following three categories of stakeholders that the included frameworks provide support for: (i) researchers, (ii) developers, and (iii) end-users including patients. Only a couple of frameworks target all three stakeholders and thereby provide a complete end-to-end solution; most target one or two. Table 2 lists stakeholders per framework and Figure 3 gives an overview of the distribution of frameworks per stakeholder category.

A researcher-or study investigator-designs mHealth studies and decides which data to collect to answer a particular research question. They are typically domain experts (e.g., psychologist) and are unlikely to have any experience in software development. This stakeholder group requires support for setting up new studies, setting up personalized interventions, fine-tuning data sampling methods and frequency, triggering surveys remotely, obtaining user consent, recruiting participants, and monitoring progress of ongoing studies. Out of the 28 published frameworks listed in Table 2, two-thirds $(N=18)$ of them target researchers. Examples of such frameworks are QuestionSys [81], EUPMS [80], Beiwe [85], AWARE [24], RADAR-base [68], and Ohmage [84]. 
Application developers use a framework to implement mHealth applications for data collection and analysis, without having to implement everything from scratch [84]. This stakeholder group expects frameworks to provide secure, modular, and extensible application programming interfaces (APIs) for both mobile phone and server-side development. Two types of frameworks targeting developers can be identified: middleware and backend frameworks. Middleware frameworks provide application programming interfaces (APIs) to build contextaware applications and pre-processing on phones, whereas back-end frameworks help with storing, analyzing, and visualizing study data on the server side. Nearly two-thirds $(N=19)$ of the 28 frameworks included in this review support developers. Besides, nearly one-third $(N=9)$ of the frameworks support both developers and researchers. All 9 open-source non-academic frameworks target developers and are intended for use in application development (see Table 2).

End-users are individuals or patients who want to use mobile devices to capture health-related data. This is typically done by self-reports (e.g., survey or questionnaires) and continuous sampling from the smartphone's built-in sensors and connected wearable devices. Such users use the sensing frameworks for use cases such as health-awareness and well-being tracking. Since they use their own phones, battery drain is a core concern for them $[24,84]$. Such frameworks typically include end-user dashboards to modify which data are collected; e.g., AWARE [24] and Funf [26] support enabling or disabling sensors. Others, such as AndWellness [32], allow endusers to specify a time when to trigger a daily survey. Although most frameworks supporting end-users allow them to view collected data to motivate them for self-reflection and behavioral improvement, only a few (e.g., Reference [32]) support instant feedback. Only Ohmage [84] provides support for privacy control by allowing end-users to delete, export, and change the privacy states of their responses and data. As shown in Figure 3, only 5 out of 28 published frameworks in this review focus on this user group, and only 2 support all three stakeholders.

\section{FUNCTIONAL FEATURES}

This section describes different functional features supported by the frameworks (RQ2). Based on thematic labeling, the functional features of each framework have been grouped into three overall categories: (i) sensing and storage, (ii) data processing and analysis, and (iii) study management. Table 2 provides an overview of all identified functional features per framework.

\subsection{Sensing and Storage}

Collection of data and storing it for later access is the primary functionality provided by most of the mHealth frameworks. This overarching feature can be further broken down into support for: (1) integration with external sensors, (2) built-in smartphone sensors and software sensing probes, (3) ecological momentary assessments (EMAs)and surveys, (4) data storage and cloud back-end, (5) context-aware sampling, and (6) remote configuration.

4.1.1 Integration with External Sensors. Wearable sensors are used in many health care studies to enable continuous monitoring of physiology parameters such as heart rate, electrocardiography (ECG), glucose level, sleep, and physical activity. For example, wearables like Fitbit [25] have been widely adopted for monitoring of health symptoms and early intervention in a clinic setting. Nearly two-thirds $(N=17)$ of the 28 frameworks in this review report support for data collection from external sensors. This is typically implemented in one of two ways: through a wired or wireless connection between the smartphone and the sensor, or by retrieving data from the device's data server, where data pre-processing, aggregation, and analysis might be done.

For wireless communication to external sensors, the majority of these frameworks used Bluetooth. Besides, few other wireless protocols such as Adaptive Network Topology (ANT+) and Bluetooth Low Energy (BLE) (in mCerebrum [35]) were also used. Only one framework, ODKSensor [15], provides a plugin interface for connecting external sensors over USB. 
Alternatively, rather than retrieving data directly from the sensors, data can be retrieved from the device's remote data repository by calling, e.g., a web API. In this scenario, sensors upload data directly to a vendor's repository either from the sensor or via a vendor-specific smartphone app. Next, vendors may perform additional data-processing on their servers to extract higher-level features that are made available through their APIs. For example, Fitbit performs advanced processing of raw accelerometer data to calculate higher-level features such as step count and sleep patterns. The drawback of this approach is that the framework and its users do not have real-time access to sensor readings, since data are only available through the web API after they have been uploaded to the server. For example, there is a delay of 15 minutes in the case of Fitbit. Additionally, such higher-level features may rely on proprietary algorithms that may change over time. Examples of this type of integration include HealthOS [48], AWARE [24], and RADAR-base [68], which provide plugins to retrieve data from sensor vendors' data repositories. In some frameworks (e.g., AWARE [24]) data collection from the sensor's server is done via the smartphone whereas in other frameworks (e.g., mCerebrum [35] and RADAR-base [68]) this happens server-to-server.

4.1.2 Built-in Smartphone Sensors and Software Sensing Probes. Modern mobile phones have a range of sophisticated built-in sensors that can sense motion, mobility, and the environment, as well as a set of network antennas and communication features, which can be used to sense social and communication behavior. A number of "mobile health sensing" studies have shown that such data can help discover correlations between various physical, behavioral, and mental health conditions [24,66]. To collect the data from the smartphone's built-in sensors and communication components, mobile sensing frameworks typically provide abstract general-purpose interfaces-often called "probes" [26]. Based on the thematic analysis, support for collecting data from built-in sensors and other components can be divided into five overall categories: (i) motion sensors, (ii) environmental sensors, (iii) communication probes, (iv) network probes, and (v) device probes. Using this categorization, Table 4 provides an overview of the different types of data that can be sensed using built-in sensors and probes on a mobile phone for each of the frameworks included in this review. Please note that these data are based on the available description as provided in the articles and code documentation (if available). It may be the case that only a few relevant sensors were mentioned. Therefore, we do not conclude that omissions in this table imply a given sensor is not supported by the framework. In some cases, articles just stated that the framework supports various built-in sensors but did not provide details. Therefore, it is likely that they might have $\checkmark$ under the "built-in smartphone sensors" category in Table 2 but missing details in Table 4.

The most commonly supported sensors mentioned are: accelerometer, GPS, gyroscope, proximity, gravity, light (ambient light intensity), magnetometer, audio, temperature, telephony (start and end time of calls), cell tower (cell towers connected to), Bluetooth (surrounding Bluetooth-enabled and visible devices), and Wi-Fi (e.g., nearby Wi-Fi access points).

Apart from the hardware sensor probes, frameworks also support software and human-based sensing probes. Such software probes include capturing data from the user's calendar, application use, emails, and the call log to capture social activity. The human-based sensing probes include simple prompting of users to input some data (e.g., label an activity for experience sampling method (ESM)) and gesture input [24]. Although some frameworks support multiple operating systems (OSs), the number of supported sensor probes varies from operating system (OS) to OS. For instance, Sensus supports SMS message and light level probes on Android but not on iOS [94]. This is partly due to different hardware setup on the different phones and OSs but also due to iOS implementing a more stringent security and privacy policy, which restricts access to users' personal data such as the phone and SMS logs.

4.1.3 Ecological Momentary Assessments (EMAs) and Surveys. Through the use of surveys, detailed observations or subjective experiences can be obtained by asking users a list of questions. Input requested from users can be in various formats: free text, radio button selection, check-boxes, Likert scale, yes/no queries, and quick responses (a simple button press). ecological momentary assessments (EMAs) involve the repeated collection 


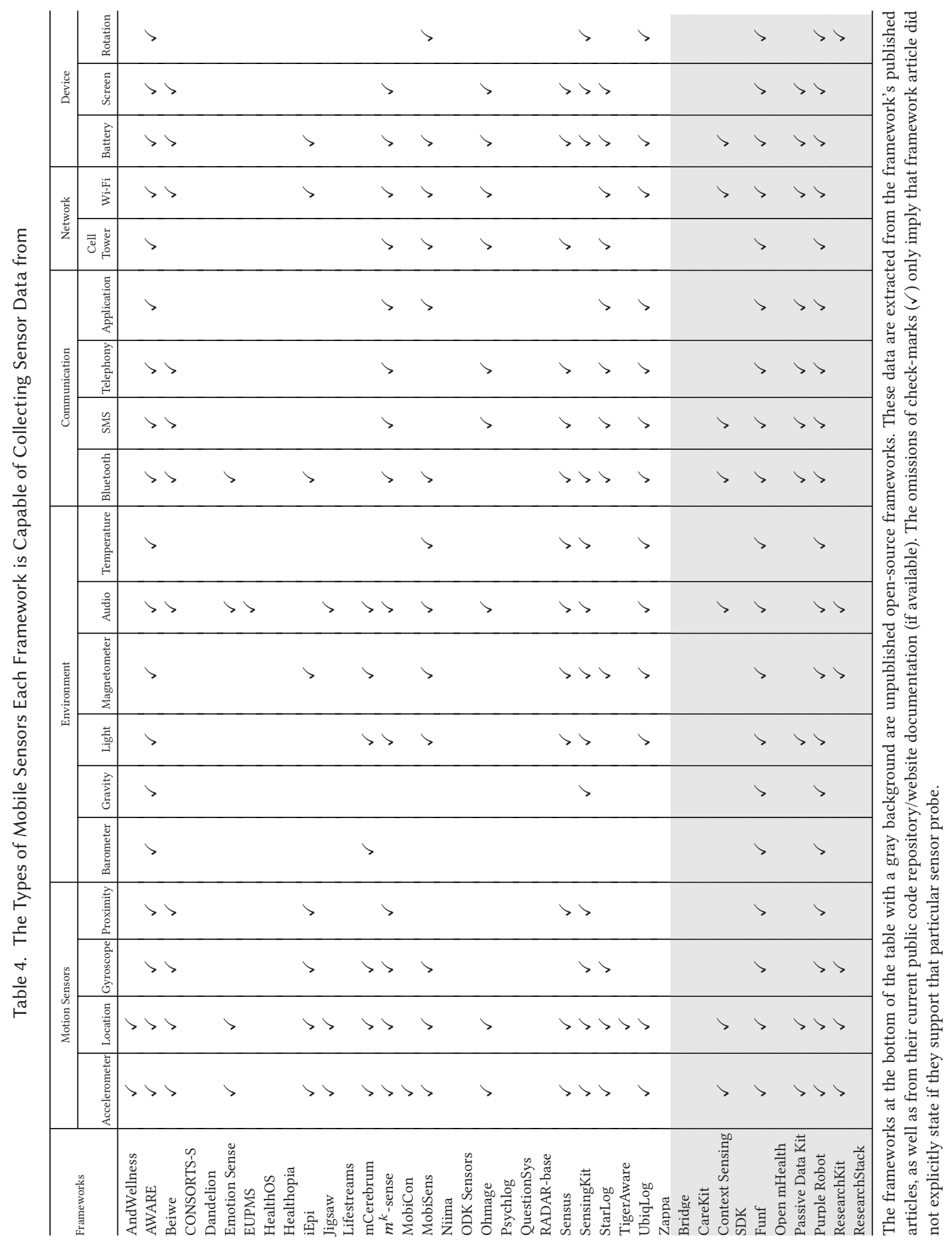

ACM Transactions on Computing for Healthcare, Vol. 2, No. 1, Article 8. Publication date: December 2020. 
of subjects' current experiences through the use of surveys in real time, in their natural setting. By prompting the user in the relevant context at the right time, EMAs help in reducing recall bias and maximizing ecological validity of the collected subjective data. Half $(N=14)$ of the 28 frameworks report support for EMAs or surveys.

Commonly reported EMA scheduling approaches are: (i) randomized, (ii) triggered at a fixed point in time, (iii) triggered by contextual events, and (iv) remotely triggered by a researcher. The selection of an appropriate EMA scheduling approach is highly use-case specific. Remote scheduling is preferred when a researcher needs to change the survey content very often. When context is important, an EMA can be triggered based on a sensed event, e.g, when the user completes 10,000 steps in a day or when the user approaches a specific geo-location.

mCerebrum [35] implements a bipartite-graph-based EMA scheduler supporting the dynamic adaptation of EMA triggers. It learns from the user's previous responses and adjusts subsequent scheduling accordingly. Sensus [94], AWARE [24], and iEpi [29] support remote and context-triggered EMA scheduling. AWARE [24] employs an EMA questionnaire-building schema defined in JSON, whereas iEpi [29] uses XML to define the content and structure of surveys. Most frameworks do not report on support for multiple survey languages. Only Ohmage [84] supports a few survey languages but not full internationalization.

4.1.4 Data Storage and Cloud Back-end. Frameworks can store the collected data locally and/or on the cloud. In the case of cloud storage, different data offloading techniques are used: when and how often data are synced to remote servers. We found that nearly half $(N=18)$ of the 28 reviewed frameworks rely on cloud storage. Across the frameworks the most common data offloading techniques were: (i) at a fixed point in time, (ii) event-based, and (iii) triggered remotely on demand.

When a fixed point in time for data offloading is chosen, data synchronization with the server takes place at regular intervals. In case the device or server is unreachable, data to be synchronized are queued locally for delivery at a later time. Event-based scheduling is a popular data offloading technique in data collection frameworks that only support low-frequency sensors or surveys. Common examples of events to schedule data offloading are: (1) when a data buffer size reaches a limit, (2) the network connectivity changes from mobile data to $\mathrm{WiFi}$, or (3) the phone is put on charge. Triggering data offloading on demand is typically used in frameworks that involve data sampling across devices. For example, in AWARE [24], the message queue telemetry transport (MQTT) protocol is used to allow devices to issue commands to other devices for data upload, exchange, and synchronization.

SQLite, an embeddable relational database management system, is used as the de facto local data storage for both Android and iOS. However, frameworks that support high-rate sensor data generate large amounts of data. Therefore, such frameworks employ several custom techniques. For instance, mCerebrum [35] implements a custom data router for offloading and efficient data sharing across different applications, called "DataKit." Similarly, RADAR-base [68] relies on Apache Kafka streams for real-time data processing and cloud storage of high volumes of incoming data.

4.1.5 Context-aware Sampling. Context-aware sampling in a sensing framework is when data collection is customized or adapted based on the user's current activity. Nearly half $(N=12)$ of the 28 frameworks report context-aware sampling support to different degrees. Different reasons for using context-aware sampling are: (i) optimizing phone resource (i.e., CPU, memory, battery) dynamics and achieving adaptability based on demands of the application and (ii) to achieve personalization in data sampling by incorporating user's preferences.

Healthopia [58] utilizes context-aware sampling to turn off a subset of sensors based on user context to reduce bandwidth usage and battery consumption (e.g., if the user is not moving then stop measuring the user's heart rate and location). mCerebrum [35], iEpi [29], and AWARE [24] use context inferred from sensors to schedule EMAs. Among the unpublished frameworks, Funf [26], Purple Robot [72], and the Context Sensing SDK [38] provide support for context-aware sampling. In particular, the Context Sensing SDK includes a context API that supports several built-in user context types such as physical activity recognition, location, environment, and 
audio classification. It also has a rule-based engine that can be used to create new rules and corresponding event triggers for optimizing phone resource.

4.1.6 Remote Configuration. Remote configuration enables researchers to remotely manage and modify a study at runtime, i.e., after it has been started. For instance, researchers can change the configuration of a running study if they observe that location needs to be sampled more frequently or the timing of an EMA needs to be changed. Only 3 of the 28 frameworks listed in Table 2 describe support for remote configuration. For the others, no description is provided in the paper. Remote configuration is most commonly supported for: (i) changing survey triggers, (ii) adapting survey content, and (iii) changing the sensors' sampling configuration. Frameworks such as AWARE [24] and $m^{k}$-sense [31] provide researchers with a web-based dashboard, which enables remote configuration. Similarly, iEpi [29] allows changing sensing and survey parameters in a running study, but does not allow changing contextual triggers for surveys. Amongst unpublished frameworks Purple Robot [72] and Funf [26] support remote configuration. Funf [26] only supports remotely changing the sensors data configuration whereas Purple Robot [72] provides a scripting engine allowing for remote configuration of both surveys and sensor configurations.

\subsection{Data Processing and Analysis}

Data processing can happen at several levels starting with simple support for data quality assessment, to support for data analysis, to extracting more high-level bio-markers, behavioral \& health features from the data.

4.2.1 Data Quality Assessment. Data quality assessment refers to the ability of a framework to assess whether or not incoming data are of acceptable quality for both passively collected sensor data and survey data. This enables warning when data are missing, sensors malfunction or need to be re-calibrated. We found that only a few $(N=4)$ of the 28 articles in our review reported on data quality assessment mechanisms. For passively collected sensor data, they were either used (i) to dynamically adjust depth and complexity of the mobile sensing process or (ii) to alert the researcher about data discrepancies, such as missing data or data outside of expected ranges. Only AndWellness [32] discusses quality assessment of survey data.

As an example, mCerebrum [35] verifies whether incoming high-frequency sensor data are of poor quality and adjusts the depth and complexity by reusing the results from other modules or by choosing an optimal classifier at runtime to achieve optimal performance. An example of supporting data discrepancy alerts is available in the iEpi framework [29], which includes a "compliance report generator" tool, including an analysis of how long each phone was actively collecting data.

4.2.2 Data Analysis. Data analysis tools enable researchers to draw insights from collected data. Depending on the use case, data processing and analysis can be performed either on the mobile phone or on the web server and during (online) or after (offline) the study completed. Nearly two-thirds $(N=19)$ of the 28 frameworks report on their support for data analysis. The granularity and approach for data analysis and processing differs from framework to framework.

The majority of articles report support for post-data-collection data processing (offline) on their back-end servers by providing custom plugins and libraries for data analysis. For instance, Lifestreams [37] is a modular mHealth data analysis stack for personal data sense-making and provides complex feature extraction such as the user's semantic location and physical activity. Similarly, SensingKit [39] describes a plug-in system that allows developers to write automated data processing scripts for pre-processing the data before extraction. Sensus [94] provides an analytics library written in R (SensusR). AWARE [24] does not have ready-made plugins for data processing and analysis, but supports building custom plugins for data processing and analysis. Among the unpublished frameworks, Bridge [11] implements "Synapse," a data analysis subsystem that periodically imports data from the main server for analysis. It creates a separate data analysis environment per individual study to prevent researchers from accessing potentially sensitive data. 
4.2.3 Bio-markers, Behavioral \& Health Features. In recent years, machine learning and deep learning models have been applied to sensor data to perform real-time behavior feature extraction, activity recognition, and develop bio-markers. mHealth researchers are utilizing such models in applications that require real-time anomaly detection (e.g., continuous stress assessment [36]) and health interventions (e.g., smoking detection [76]). These deep learning-based digital bio-marker models can facilitate new value chains when applied to longitudinal mHealth data, thus help in discovering new behavioral patterns and the personalization of healthcare.

Twelve out of the 28 frameworks support models for bio-markers and health and/or behavior feature extraction. Some prominent supported bio-marker or health feature models are abnormal heart-rate detection [35, 45, $49,58,77]$, fall detection [77], and activity recognition [37, 45, 51, 92]. Among others, mCerebrum [35] has an emphasis on supporting reusable bio-markers and provides models for detecting events such as stress, smoking, and eating. Its architecture enables the creation of study specific bio-marker modules that may build on top of existing ones.

\subsection{Study Management}

Study management involves handling (1) participant consent and (2) study setup and monitoring.

4.3.1 Participant Consent. When conducting an mHealth study, obtaining participant consent is essential. The researcher must ensure that study participants are informed about which data are collected, who can access them, and for what purpose they will be used. None of the published frameworks listed in Table 2 provide support for obtaining participant consent, and consent is typically implemented in a study-specific application or obtained in a paper-based form. Among the unpublished frameworks, however, three frameworks [11, 70, 71] provide support for participant consent. Bridge [11] supports defining how consent should be obtained and what it should be obtained for as part of configuring a study protocol, including information such as signature, the scope of data sharing (who all can access data) and consent signing and withdrawal dates. If the consent configuration gets updated during the course of a study, the participant is notified. Likewise, both ResearchKit [70] and ResearchStack [71] provide user interface templates for consent documents with predefined sections such as an overview of the study, information on how data are gathered, privacy policies, how the collected data will be used, time commitment, and consent withdrawal information. Custom study-specific consent sections can be added if needed. Overall, this saves development time.

4.3.2 Study Setup and Monitoring. Nearly one-third $(N=9)$ of the 28 frameworks listed in Table 2 provide support for study setup and monitoring through a user interface, typically through a web portal. This includes support for: (i) study participant recruitment, (ii) creating and scheduling surveys, (iii) visualizing study progress, and (iv) remote configuration of sensors and survey triggers.

All the frameworks that support study setup and monitoring provide a dashboard for the researchers to visualize the collected data. Besides this, frameworks such as AWARE [24], Beiwe [85], and Sensus [94] provide assistance in registering study participants, creating, editing, and deploying surveys. AWARE [24] additionally supports editing sensor sampling configurations, changing the database, adding co-researchers, and viewing the devices linked to a study. In addition to providing researchers access to collected data, two frameworksOhmage [84] and Andwellness [32]-also enable study participants to view their data. Ohmage [84] in particular further allows the participant to change their privacy settings, delete, and export their study data.

\section{NON-FUNCTIONAL FEATURES}

This section presents the non-functional features of the frameworks and hence addresses the second part of RQ2. In traditional software engineering, a non-functional requirement (NFR) is defined as "a software requirement that describes not what the software will do but how the software will do it" [1]. non-functional requirements (NFRs) are used to evaluate the operation of a system, rather than specifying its functional behavior. For the 28 
published frameworks, we looked for NFR details in the papers. For the unpublished frameworks, we looked at information on their respective websites, but overall very limited information was available. Please note that in case no details on an NFR are presented, this does not necessarily mean it is not addressed by the framework, but simply that we could not find any mention of it in the screened sources. The review focused on four categories of NFRs, which are most relevant to mobile sensing frameworks: (i) extensibility, (ii) scalability, (iii) security and privacy, and (iv) license and documentation. An overview of all identified NFRs per framework is provided in Table 5.

\subsection{Extensibility}

In software engineering, extensibility denotes the ability to add, enhance, or repair existing functionality in a system or component [19]. Since the number of available built-in sensors in modern smartphones keeps increasing and new wearable sensors emerge continuously, it is important that these can easily be integrated into existing sensing frameworks. For extensibility, we checked if the framework article describes an APIs for adding new functionalities, including support for adding new sensing capabilities. More than two-thirds of the articles $(N=19)$ state that they support extensibility. It is interesting to note that a total of 10 articles just state support for extensibility without providing any further details. In the articles describing the extensibility mechanisms, there were typically two ways to extend the framework: (i) an API for adding new smartphone sensing modalities (such as a light sensor on the phone) and/or data types to be collected or (ii) a plugin mechanisms for adding support for external sensors (like an electrocardiography (ECG) monitor).

Examples of frameworks supporting the first type of extensibility include AndWellness [32], AWARE [24], Passive Data Kit [82], Funf [26], and Purple Robot [72], which all support extensibility and modifiability by providing an interface to add new custom smartphone sensor probes. Extensibility can also be supported on the back-end of a framework, where extensibility focuses on the ability to support new data types of custom data sources. For instance, Ohmage [84] implements a backward-compatible API to achieve extensibility. However, Open mHealth [57] allows adding new custom data schemas and also provides an extension for integration with external data sources such as HealthKit and data collected from electronic health records (EHR)s.

In the second category, we identified frameworks such as AWARE [24], UbiqLog [69], and HealthOS [48], which support extension of the framework to collect data from external sensors. ODK Sensors [15] supports extensibility by providing an interface between external sensors and a smartphone for managing device discovery, data buffers, and communication channels. ODK Sensors also provides an ecosystem of underlying reusable sensor drivers that enables developers to write minimal sensor-specific code.

\subsection{Scalability}

In software engineering, scalability is a desirable property for a computing system to have. And while the basic notion is intuitive, scalability has no generally accepted definition [33]. Therefore, in this review, we examined how scalability is described in the articles or websites of the included frameworks. Of all the frameworks listed in Table 5, more than two-thirds $(N=19)$ of the articles mention support for scalability. However, out of these, only 7 present additional details to back up the claim that the presented framework is scalable. For each framework that provided details on scalability, we investigated whether support for scalability was (i) argued based on the underlying technology used or (ii) claimed based on a dedicated scalability evaluation.

In two articles, the authors argue for the scalability of the framework based on a description of the underlying infrastructure and technology used to implement the framework. Ranjan et al. [68] state that RADAR-base relies on the Apache Kafka platform [20] as the underlying infrastructure to achieve scalability. However, no scalability evaluation is reported. In AndWellness [32], the authors state that they rely on the Spring framework for server scalability, as it provides component replaceability and flexibility.

Some articles report on scalability evaluations. Examples include mCerebrum [35], AWARE [24], and Ohmage [84]. The article on mCerebrum [35] describes a high-frequency sensor data collection test on the phone, the results of which indicate that the framework outperforms others, such as GoogleFit [28], AWARE [24], 
Table 5. Non-functional Features

\begin{tabular}{|c|c|c|c|c|c|c|c|}
\hline Framework & Extensible & Scalability & & Security & Privacy & Open & Docs \\
\hline AndWellness & $\checkmark$ & $\checkmark$ & $\checkmark$ & DENC,SPC & $\checkmark$ & & \\
\hline AWARE & $\checkmark$ & $\checkmark$ & $\checkmark$ & DENC,SPC & $\checkmark$ & $\checkmark$ & $\checkmark$ \\
\hline Beiwe & $\checkmark$ & $\checkmark$ & $\checkmark$ & DENC,EXT,SPC & $\checkmark$ & $\checkmark$ & $\checkmark$ \\
\hline CONSORTS-S & $\checkmark$ & & & & & & \\
\hline Dandelion & $\checkmark$ & & & & & & \\
\hline Emotion Sense & & $\checkmark$ & & & & $\checkmark$ & $\checkmark$ \\
\hline EUPMS & & & $\checkmark$ & DENC & & & \\
\hline HealthOS & $\checkmark$ & $\checkmark$ & $\checkmark$ & DENC & $\checkmark$ & & \\
\hline Healthopia & & & & & $\checkmark$ & & \\
\hline iEpi & & $\checkmark$ & $\checkmark$ & DENC & $\checkmark$ & & \\
\hline Jigsaw & $\checkmark$ & $\checkmark$ & & & & & \\
\hline Lifestreams & $\checkmark$ & & & & $\checkmark$ & $\checkmark$ & $\checkmark$ \\
\hline mCerebrum & $\checkmark$ & $\checkmark$ & $\checkmark$ & DNA & $\checkmark$ & $\checkmark$ & $\checkmark$ \\
\hline MobiCon & $\checkmark$ & & & & & & \\
\hline MobiSens & & & & & $\checkmark$ & & \\
\hline$m^{k}$-sense & & $\checkmark$ & $\checkmark$ & DENC & $\checkmark$ & & \\
\hline Niima & $\checkmark$ & $\checkmark$ & $\checkmark$ & DENC,SPC & $\checkmark$ & & \\
\hline QuestionSys & & $\checkmark$ & & & & & \\
\hline ODK Sensors & $\checkmark$ & $\checkmark$ & & & & & \\
\hline Ohmage & $\checkmark$ & $\checkmark$ & $\checkmark$ & DNA & $\checkmark$ & $\checkmark$ & $\checkmark$ \\
\hline Psychlog & & & & & & $\checkmark$ & \\
\hline RADAR-base & $\checkmark$ & $\checkmark$ & $\checkmark$ & DENC & $\checkmark$ & $\checkmark$ & $\checkmark$ \\
\hline Sensus & & $\checkmark$ & $\checkmark$ & EXT & $\checkmark$ & $\checkmark$ & $\checkmark$ \\
\hline SensingKit & $\checkmark$ & & & & & $\checkmark$ & $\checkmark$ \\
\hline StarLog & & & & & $\checkmark$ & & \\
\hline TigerAware & $\checkmark$ & $\checkmark$ & $\checkmark$ & DENC & $\checkmark$ & & \\
\hline UbiqLog & $\checkmark$ & $\checkmark$ & $\checkmark$ & DENC,SPC & $\checkmark$ & $\checkmark$ & \\
\hline Zappa & $\checkmark$ & $\checkmark$ & & & & $\checkmark$ & \\
\hline Bridge & $\checkmark$ & $\checkmark$ & $\checkmark$ & DENC,SPC,EXT & $\checkmark$ & $\checkmark$ & $\checkmark$ \\
\hline CareKit & $\checkmark$ & $\checkmark$ & & & & $\checkmark$ & $\checkmark$ \\
\hline Context Sensing SDK & $\checkmark$ & & & & $\checkmark$ & & $\checkmark$ \\
\hline Funf & $\checkmark$ & $\checkmark$ & $\checkmark$ & DENC & $\checkmark$ & $\checkmark$ & $\checkmark$ \\
\hline Open mHealth & $\checkmark$ & $\checkmark$ & $\checkmark$ & SPC & & $\checkmark$ & $\checkmark$ \\
\hline Passive Data Kit & $\checkmark$ & & & & & $\checkmark$ & \\
\hline Purple Robot & $\checkmark$ & & & & & $\checkmark$ & \\
\hline ResearchKit & $\checkmark$ & $\checkmark$ & & & & $\checkmark$ & $\checkmark$ \\
\hline ResearchStack & $\checkmark$ & $\checkmark$ & $\checkmark$ & DENC & & $\checkmark$ & $\checkmark$ \\
\hline
\end{tabular}

The frameworks at the bottom of the table with a gray background are unpublished open-source frameworks. Check marks $(\checkmark)$ indicate that the article provides a description of the listed non-functional feature. Open $=$ Open Source, SPC $=$ Secure protocol, EXT $=$ Rely on external infrastructure for security, DENC $=$ Data encryption, DNA $=$ Details not available (the framework states that it has support for security, but details of the security mechanisms are not specified). 
and HealthKit [30]. Other articles report on scalability tests on the server-side. For example, the Ohmage [84] server was load-tested using over a million API calls, and scalability of the AWARE [24] server dashboard was demonstrated by increasing the number of participants and measuring response time for page load, visualization, sorting, and searching.

\subsection{Security and Privacy}

Since sensitive health and behavioral data are collected as part of mHealth studies, support for security and privacy in sensing frameworks is essential. In this review, we have investigated which techniques the frameworks use to ensure data security as well as privacy.

5.3.1 Security. Surprisingly, we found that only half $(N=14)$ of the 28 articles discuss security techniques. The main security techniques presented are (i) data encryption (DENC) and (ii) data transfer over secure protocols (SPCs).

Data encryption is a security method that allows data encoding that then can be only decoded with the correct encryption key. A total of 11 articles in this review report using encryption for security, and five out of these stated using Advanced encryption standard (AES). Advanced Encryption Standard (AES) is encryption specification set by United States' National Institute of Standards and Technology [63] for encryption of electronic data. AWARE [24], Beiwe [85], and Ohmage [84] are examples of frameworks that use AES. Among the unpublished frameworks, three frameworks use encryption: Funf [26], Bridge [11], and ResearchStack [71].

All frameworks that provide details on how they securely transfer data to the server (SPC) do so by using Secure Sockets Layer (SSL). Among unpublished frameworks, two frameworks-mHealth [57] and Bionetworks [11]-use Secure Sockets Layer (SSL). In addition, there are three frameworks in which the authors state that they provide secure data collection and offloading, but do not provide any additional details (labeled as "DNA" in Table 5).

Three frameworks-Sensus [94], Beiwe [85], and Bridge [11]-report that they rely on the security mechanisms of a cloud provider (e.g., Amazon Web Service (AWS)) for back-end data security (labeled as "EXT" in Table 5).

5.3.2 Privacy. Collecting contextual and physiological data in mHealth sensing involves significant privacy risks. Therefore, it is relevant for mHealth sensing frameworks to implement privacy-preserving techniques as part of data collection, processing, and sharing. We found that 17 of the 28 articles discuss privacy-preserving techniques. The key techniques used are: (i) deleting personally identifiable information, (ii) one-way hashing, and (iii) storing personal information and sensor data on separate servers.

One-way hashing is a technique that converts a message into a unique message digest in an irreversible way. It is often used to obfuscate and protect sensitive information. Three frameworks $[5,24,85]$ use one-way hashing to obfuscate personal identifiers (e.g., phone numbers and MAC address). Beiwe [85] uses the standard SHA-256 algorithm for hashing phone numbers, whereas Niima [5] uses a two-stage hashing process in which the first hashing takes place on device and the second hashing is done on the server using a secret Salted Hash [40] algorithm. One framework-RADAR-base [68]-keeps sensor data and user information on separate servers to achieve pseudonymization. mCerebrum [35] introduced a new privacy preservation technique by means of implementing central privacy and access controllers. Its central privacy controller allowed participants to suspend sensor specific data collection and sharing.

Among the unpublished frameworks, both ResearchKit [70] and ResearchStack [71] provide means to list privacy policies in the user consent module. However, the implementation details of the specific privacy technique is left to the individual applications that are built using these frameworks. Two others-Funf [26] and Bridge [11]-use one-way hashing for anonymization. 


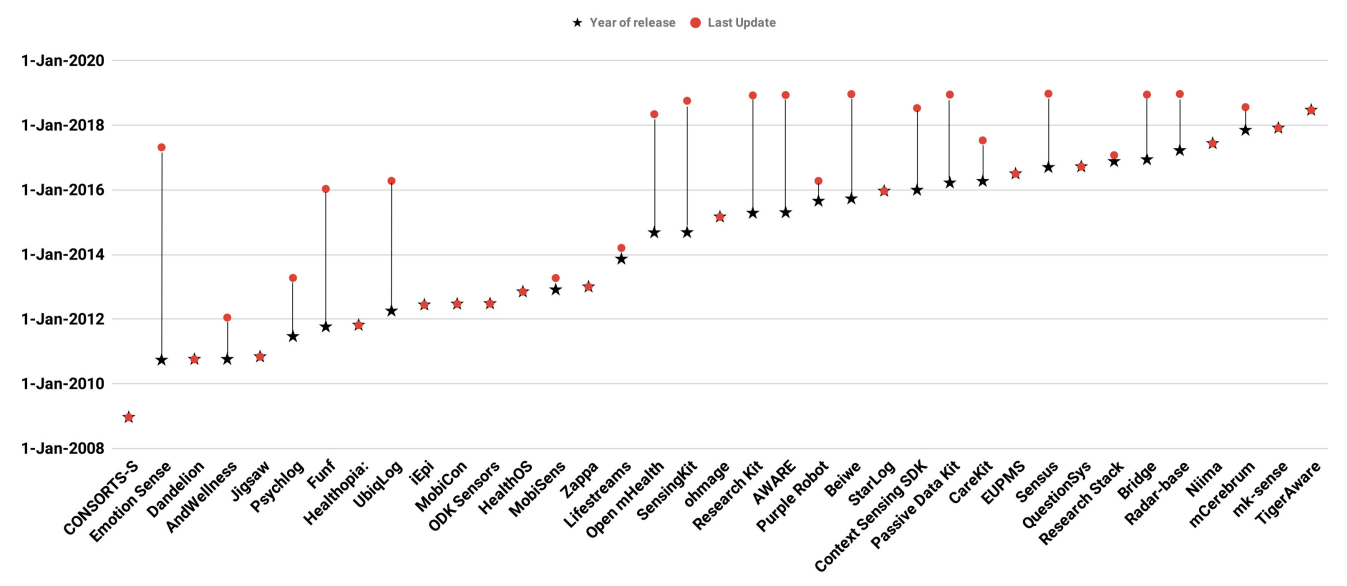

Fig. 4. Initial release and last update time for all frameworks.

\subsection{License Model and Documentation}

To make sensing frameworks reusable, maintainable, and extendable, detailed and accurate documentation is necessary. Moreover the software license model is important for developers and researchers to consider when choosing a framework, since copyright restrictions could limit how the framework can be used and modified. To obtain information about the license and available documentation, we looked at framework websites and their code repositories in the case of open-source frameworks.

5.4.1 License Model. Over one-third $(N=12)$ of the 28 frameworks in this review are open source. Seven of them are licensed under the Apache License 2.0, three under BSD 2-Clause "simplified," one under BSD licenses, and one under GNU Lesser GPL v3.0 (LGPL). All the unpublished frameworks except for Context-Sensing SDK [38] are open source: five are licensed under the Apache License 2.0., two under BSD License, and one under GPL v3.0.

5.4.2 Documentation. Since it is difficult to assess whether a framework is sufficiently documented, we only looked at whether documentation of any kind, be it on an architectural level or API level, is provided. We found that less than one-third $(\mathrm{N}=9)$ of the 28 frameworks provide some level of documentation. In contrast, all of the unpublished (mainly commercial) frameworks provide detailed API documentation.

\section{MAINTENANCE}

The main purpose of researching, designing, and implementing a mHealth framework is to support re-usability of core components and technology, thereby enabling easier creation of mHealth applications. This kind of reusability and cross-application technology requires, however, that the frameworks are updated and maintained on a regular basis to accommodate changes in the underlying OS and to support new features. This section investigates the maintenance of the frameworks, i.e., research question 4 (RQ4). We are interested in understanding how the identified mHealth sensing frameworks have evolved over time and to what degree the frameworks are being updated and maintained after their initial release.

To identify the period over which frameworks have been maintained, we compare the first and last date at which information about the framework has been made available, illustrated in Figure 4. Concretely, for the first date, we used the publication date of the framework's article or the date of the first release of the software for non-published frameworks. For the last date, we chose the date of the last commit to the framework's public code repository or alternatively the date of the last listed release on the corresponding website. We found that more than two-thirds $(N=21)$ of the included frameworks (including the unpublished frameworks) maintained 
their repositories after the initial release. Ten frameworks have been updated in the year 2018 (the time this review was concluded). AWARE [24], SensingKit [39], Sensus [94], RADAR-base [68], Beiwe [85], Bridge [11], and mCerebrum [35] are some of the most regularly updated frameworks that were still being maintained in 2018. The past few years (2015-2018) have seen a dramatic increase in the number of released frameworks. Also notable is that up until 2014 most frameworks were middleware libraries, whereas most recent frameworks (in or after 2015) are end-to-end mHealth frameworks that include support for sensing, storage, processing, visualization, and study management.

\section{DISCUSSION}

This review has shown that, since 2010, dedicated research into mobile and wearable sensing frameworks for mHealth has resulted in a non-trivial set of sensing technologies. Approximately ten frameworks are maintained today (see Figure 4) and the more recent ones are far more comprehensive, including sophisticated functional and non-functional features on both the phone and the server side of the technology (see Tables 2 and 5). In addition to the early frameworks that were mainly driven by academic researchers there has been a growing interest in mHealth from industry. Several commercial frameworks, such as HealthKit [30] and GoogleFit [28], have been introduced to support the creation of mHealth sensing applications in the Apple and Google technology ecosystems, respectively.

In terms of functional features, several recent frameworks have focused on functionalities such as remote configuration and study monitoring support (see Table 2), which enable researchers to change data sampling configuration, survey content, and triggers remotely. Also, given the advancement in machine learning and deep learning, support for features such as behavioral and health-biomarkers computation is starting to be included in recent frameworks [35]. Due to limited resources on the smartphone (e.g., computational power, battery, and network bandwidth), the initial work on feature extraction and data analysis was mostly done offline on servers. Adding support for high-level feature extraction on smartphones has the potential of enabling the design of advanced just-in-time interventions in health sensing applications.

The unpublished open-source frameworks seem more popular, gauged by the number of people that starred their code repositories. This might be due to the fact that these frameworks typically come with good documentation, including tutorials, and are maintained by a professional software development team of a large company. Amongst the research-based frameworks, AWARE [24], Sensus [94], and Beiwe [85] seem to be the most popular in terms of people that starred their repositories.

The majority of framework evaluations focused on system resource management and impact on battery life while sensing, storing, and transferring data over the network. However, the evaluations did not use a common or standardized way to evaluate performance and efficiency. For example, different versions of smartphones from different manufactures were used. This complicates comparing efficiency and performance across frameworks. Nevertheless, there are some recurring findings. Evaluations of frameworks such as StarLog [59], AndWellness [32], SensingKit [39], and Aware [24] show that configuring high sampling frequencies for sensing probes is associated with draining the battery very rapidly. But, there are ways to mitigate this. An evaluation of mCerebrum's micro-batching strategy showed a significant reduction in CPU use while ingesting high-frequency sensor data. Thus, this makes micro-batching a good strategy for applications that require real-time data sharing and processing. In addition, none of the reviewed frameworks reported on health efficacy of applications built and deployed using them. They solely report on technical proof-of-concept studies within different health domains. But, it must be noted that such studies are typically reported in separate papers, referring back to the toolkit/framework papers, which were not included in this review.

\subsection{Implications on Healthcare Research}

There is mounting evidence that mHealth applications are practical and low-cost means to deliver disease prevention, self-management, diagnostics, and treatment based on commodity hardware. A simple app could give 
anyone, anywhere, the ability to perform diagnostic screening tests and monitor their condition after they receive an official diagnosis [53]. Moreover, healthcare may become more available and affordable in resource-poor settings in a way that was previously unimaginable $[4,22]$. The reviewed frameworks enable the creation of such mHealth apps in a manner in which developers and researchers can more easily design, implement, deploy, and monitor mHealth applications and studies for a growing number of diseases and user groups. As shown in Table 3 and Figure 3, the reviewed frameworks have been utilized for building a diverse range of mHealth applications for different stakeholders.

However, the review also points to a set of issues in this line of research. First, despite that a major part of the frameworks were designed for mHealth application development and targeted developers as their primary stakeholders, we found that such frameworks were actually only used in a limited (1-3) number of mHealth applications. And, they are rarely used by researchers outside of the group of researchers who implemented the framework in the first place. Hence, it seems that the overall goal of having application developers adopt reusable mHealth frameworks has still not been achieved.

Second, from a healthcare perspective, the use of detailed data sampling from mobile and wearable devices still needs to fit into the overall clinical treatment and care of diseases. Many of the application studies reported are still early work and basic research, trying to understand the role and utility of mHealth sensing. Much of the research so far has targeted sensing from the mobile phone-such as accelerometer, movement, and phone interaction-that reveals information about the patient's behavior but very little about the patient's health status. Very limited work has been addressing general-purpose collection of health-related data such as physiological signals, bio-chemical data, and medication. A few frameworks-like HealthOS [48]-provide custom adapters to medical wearable devices, but their adapters are not generic and are limited to a handful of consumer health devices (i.e., not medical grade). Moreover, due to the voluminous size of data and limited time of researchers, not all insights that could be obtained from the collected data are found [17, 89]. Thus, there is a need for automatic detection of higher-level biomarkers for these frameworks to be useful in the clinic. Hence, from a healthcare perspective, multi-parametric data collection as well as the recognition of higher-level biomarkers will be needed.

Third, the frameworks available now are quite mature and seem to be maintained and backed by larger groups or consortiums of researchers, rather than individuals. This is promising for the use of these frameworks in the design and development of mHealth applications for clinical use. However, the main focus of current frameworks is on data collection, which is useful for population screening and disease diagnosis and monitoring. But, there is little or no support for interventions and delivering treatments. Examples of such treatment components could be targeting educational material, medication management including reminders, behavioral or cognitive therapy, and recommendations for healthy living. As such, the flow of data and information in these frameworks seems to be "one way," i.e., flowing from the patient to the clinicians, while little or no information flows in the other direction. Some of the frameworks are, however, designed to be used in conjunction with other frameworks, which may support interventions. But for the majority of the frameworks this is not the case.

Nevertheless, for healthcare research, the use of such data sampling frameworks may open doors for largescale data analysis in healthcare. "Big data" and cross-study analysis can help researchers in unearthing new trends in disease management and treatments at both the individual and population level. Also, through standardized and exportable study protocols (sampling profile, data analysis tools, survey, and EMAs configurations, etc.) frameworks could significantly enhance study reproducibility, replicability, and transparency in mHealth research.

The selection of a framework for research or development purposes should be done with care. We hope this review can help guide healthcare researchers and/or mHealth application developers in the selection of a framework that suits their needs. 


\subsection{Research Gaps and Recommendations for Future Research}

This review has shown that several mHealth sensing frameworks exist, some of which are maintained and are fairly popular in terms of use and offer quite an advanced set of functional and non-functional features. As such, research into mHealth sensing frameworks seems to have come a long way, and several frameworks are available to choose from for researchers and application developers. A relevant question is then whether there are still open research questions to be addressed. Based on our review, we have identified six gaps and thus opportunities for further research. This section discusses these gaps and provides recommendations for future mHealth frameworks.

7.2.1 Personalizing Data Collection. Frameworks suffer from poor power management and cloud offloading strategies [35, 92]. In most frameworks, sampling frequencies, data processing, and data offloading techniques are either hard-coded or one configuration is provided for all participants. The existing frameworks do not take individuals' preferences, mobile hardware, resource availability, and so on, into account. Such non-personalized data collection results in poor resource management, which can be expected to annoy users of longitudinal mHealth studies. Even though frameworks such as AWARE [24] and Beiwe [85] support remote re-configuration of sampling probes after the deployment of a study, this reconfiguration needs to be done manually and cannot be personalized to individual participants. We argue that resource management and therefore user experience in mHealth applications can be improved by incorporating adaptive personalization and dynamic re-calibration of sampling profiles. This could include the use of various code offloading techniques-techniques that dynamically migrate processor-intensive tasks to cloud surrogates.

7.2.2 Data Standardization. The frameworks described in this review support data collection from a wide range of sensors. These data, however, are not stored in any standardized format and none of the frameworks that do sensing support specifying standardized data schemes to use for sampled data. This implies, for example, that blood glucose measurements collected from the same device but via two different frameworks cannot be directly compared, as there is no common standard for storing blood glucose (including the context within which the measurement was collected). Similarly, since patient-generated data do not comply to any clinical standards, these data are hard to integrate into existing clinical systems, limiting its use [89]. Furthermore, to allow for reusable higher-order features and bio-markers extraction, and for cross-device and cross-study analysis, it is essential that all the data points for a specific measure (e.g., heart rate or blood glucose) are represented in a standardized format, regardless of the framework and/or devices from which the data were collected.

Currently, there are some initiatives to standardize mHealth data. The Health Level 7 (HL7) standardization initiative has a "Mobile Health" work group, which creates and promotes health information technology standards and frameworks for mobile health [34]. The Institute of Electrical and Electronics Engineers (IEEE) develops and maintains the IEEE 11073 standards on "Personal Health Devices' [61] as well as the P1752 open standard for Mobile Health Data [62], which is based on the Open mHealth initiative [57]. However, none of the reviewed frameworks (except Open mHealth of course) supports any of these standards. Therefore, this could be a topic for the evolution of mHealth frameworks.

7.2.3 Measurable User Privacy Support. Mobile and wearable sensing frameworks provide a novel opportunity for long-term health sensing. However, due to the sensitive nature of health data, such frameworks pose a risk to users' privacy, which is important to consider, especially given the recent introduction of the General Data Protection Regulation (GDPR) in Europe. Even though several frameworks such as mCerebrum [35] and AWARE [24] have implemented various privacy protection mechanisms (e.g., pseudonymization; central privacy and access controllers), they are mainly focused on privacy protection as part of data collection. But, when sharing data across different studies to enable "big data" analysis, additional concerns arise. Data should not be used for a different purpose than the user has given consent for. Similar to security and encryption standards, 
incorporating privacy compliance standards (e.g., BS 10012:2017 [23]) in mHealth frameworks could help in achieving measurable user privacy.

7.2.4 Measurable User Feedback. We observed that out of their three target audiences, namely, developer, researcher, and end-user, frameworks in this review mainly reported on the end-user's feedback and usability evaluation. Except for a few (e.g., EUPMS [80]), there was no reporting on feedback from developers and researchers who used these frameworks in various applications. Alongside the technical evaluations, reporting the measurable feedback from all the stakeholders will help others to identify which framework is suitable for them.

7.2.5 User Consent Support. When enrolling participants in a research study, user consent needs to be obtained. By giving consent, users give approval to be part of a study and for researchers to collect and process data for the specified purposes. Traditionally, such consent is given on paper by signing an informed consent document after having read a detailed description of the study or the purpose of the application. The introduction of new regulations such as General Data Protection Regulation (GDPR) [18] are empowering users to have full control over their data during and after the study is done, and puts forth the need for advanced consent management and configuration. ResearchKit [70] provides support for showing study information to the user on a phone and having him or her sign a consent form in a user-friendly manner. However, ResearchKit does not in any way support additional support for handling this consent during or after a study is done. Except for Bridge [11], all other frameworks in this review supporting the registration of consent leave further consent handling to the individual application that has been built using that framework. In addition, none of the frameworks provide support for handling partial consent or withdrawal of consent.

If user consent is not handled at the framework level, guaranteeing compliance with given consent during data sharing and processing within a framework may be challenging. A researcher might use data for another purpose than for which consent was given. Or, consent may expire, be modified, or retracted entirely. Manual tracking of consent forms and modifications made to them in a longitudinal study is challenging and negligence might pose privacy and legal risks. Providing support for obtaining and maintaining user consent in mHealth frameworks can help researchers identify which part of collected data the user has consented to and for which parts consent was withdrawn or modified. This would help in building more granular access restrictions for data processing and sharing.

7.2.6 Support for Study Reproducibility. When sensing frameworks are used to build applications for clinical and biomedical studies, support for study reproducibility becomes important [85]. The frameworks in this review offer limited or no support for study reproducibility. There have been some initiatives in this direction by Beiwe [85], which stores a configuration file specifying all of the applications settings to reproduce the study, such as surveys, user prompts, sensor settings, and version of the data analysis tool used. By standardizing data collection and data analysis, mHealth frameworks can help achieve study reproducibility and thereby encourage replication studies. For example, they could support exporting study protocols that define the study's technical know-how such as configuration and specifications of sensors and devices used, data sampling profile, defining when data should be collected, which stimuli need to be presented, tasks, interventions, and how user consent should be obtained for the experiment. The same study protocol could then be used by other researchers who wish to replicate the study.

7.2.7 Internationalization of Surveys. In software engineering, internationalization refers to tasks and activities by which a software system can adapt to different languages, cultural, and regional requirements without programmatic modifications (code) [93]. With the growing availability of smartphones, mHealth studies and interventions are reaching far beyond the English-speaking world. Therefore, it is essential for sensing frameworks to support various languages, text, and data input formats to reach all potential users. Only one framework (Ohmage [84]) in this review supports multiple languages for survey questions. However, it still does not support 
full internationalization. Achieving full support for internationalization requires incorporating best practices such as string externalization, full support for Unicode, standard resource file types, and locale and culture awareness (e.g., date and time format, calendar differences) into the architecture of the sensing framework.

7.2.8 Gap between Collected mHealth Data and Clinical Knowledge. As discussed earlier, the reviewed frameworks focus primarily on supporting data collection. However, there is a big gap between collecting mHealth data and utilizing it according to clinical knowledge, such as incorporating it into workflow, decision-making, and so on, which has not been addressed in any of the reviewed frameworks. Previous studies have shown that in clinical settings getting actionable info from collected mHealth data remains a challenge due to limited time and resources [21,89]. Therefore, future frameworks should also focus on bridging the gap between collecting information and obtaining actionable clinical knowledge from it.

\subsection{Threats to Validity}

There are two potential threats to validity that may affect our findings: the method adopted for selecting articles and unavailable data during data extraction.

7.3.1 Selecting Articles. As presented in Section 2, our review focuses on frameworks that may be used for health or behavior sensing. Some relevant articles may omit a discussion of the applicability of the presented framework in this context, in which case it would not show up in our search results. In addition, many of the data collection frameworks are published as part of a study. In such cases the main focus of the article is on the study and not the sensing framework. Thus, judging them solely on the basis of the title, it is likely that frameworks, which are described as part of a study article, might have been left out.

7.3.2 Unavailable Data. Since many articles do not explicitly report on all available functional and nonfunctional features, it is not always possible to assess whether or not a certain framework supports a given feature. Authors might omit mentioning certain features when they are already present in prior work, since they want to focus on novel contributions instead. Hence, we can only report on whether an article explicitly describes a particular feature. Therefore, features in the presented tables that do not include a check mark for a particular framework indicate the given feature was not discussed in the paper, but does not rule out entirely that it is not supported.

\section{CONCLUSION}

Generic sensing frameworks facilitate the development and deployment of mHealth applications and relieve developers and researchers from worrying about the underlying issues pertaining to smartphone and wearable sensor data collection, data offloading, processing, analysis, visualization, feature extraction, phone resource optimization, and study management. As the number of publications over time in this review indicate, research into the design and implementation of mHealth frameworks has grown in recent years. More recent frameworks include support for more and more functional and non-functional features. In this systematic literature review (SLR), we identified a list of 28 published and 9 unpublished open-source mHealth frameworks. Nearly one-third $(\mathrm{N}=11)$ of the reviewed frameworks are still actively maintained.

We classified the functional features they support in three broad categories: sensing and storage, data processing and analysis, and study management. There are a total of 12 frameworks that support functional features in all three categories. With regard to non-functional features, our results suggest that there has not been enough emphasis on non-functional features in mHealth sensing frameworks and a large number of the articles just state supporting non-functional features without providing additional details such as necessary tradeoffs to make or a performance evaluation.

Based on this review, we discussed the state-of-the-art in mHealth sensing frameworks and identified new features that are relevant to explore in future research on such frameworks. We hope this overview of functional 
and non-functional features, health studies in which existing frameworks have been used, how well frameworks have been maintained, and which license they use can help researchers and developers decide which framework is suitable for their next mHealth application.

\section{ACKNOWLEDGMENTS}

The authors would like to thank the anonymous reviewers and the editor for their very valuable feedback on earlier drafts of the paper.

\section{REFERENCES}

[1] Kevin MacG Adams et al. 2015. Nonfunctional Requirements in Systems Analysis and Design. Vol. 28. Springer.

[2] Sasan Adibi. 2015. Mobile Health: A Technology Road Map. Vol. 5. Springer.

[3] Nadav Aharony, Wei Pan, Cory Ip, Inas Khayal, and Alex Pentland. 2011. Social fMRI: Investigating and shaping social mechanisms in the real world. Pervas. Mob. Comput. 7, 6 (2011), 643-659.

[4] Shahriar Akter and Pradeep Ray. 2010. mHealth-An ultimate platform to serve the unserved. Yearb. Med. Inf. 19, 01 (2010), 94-100.

[5] Talayeh Aledavood, Ana Maria Triana Hoyos, Tuomas Alakörkkö, Kimmo Kaski, Jari Saramäki, Erkki Isometsä, and Richard K. Darst. 2017. Data collection for mental health studies through digital platforms: Requirements and design of a prototype. FMIR Res. Protoc. 6, 6 (2017), e110.

[6] Apple. 2016. CareKit. Retrieved March 2, 2019 from http://carekit.org.

[7] Joost Asselbergs, Jeroen Ruwaard, Michal Ejdys, Niels Schrader, Marit Sijbrandij, and Heleen Riper. 2016. Mobile phone-based unobtrusive ecological momentary assessment of day-to-day mood: An explorative study. F. Med. Internet Res. 18, 3 (2016), e72.

[8] Sangwon Bae, Tammy Chung, Denzil Ferreira, Anind K. Dey, and Brian Suffoletto. 2018. Mobile phone sensors and supervised machine learning to identify alcohol use events in young adults: Implications for just-in-time adaptive interventions. Addict. Behav. 83 (2018), $42-47$.

[9] Sangwon Bae, Denzil Ferreira, Brian Suffoletto, Juan C. Puyana, Ryan Kurtz, Tammy Chung, and Anind K. Dey. 2017. Detecting drinking episodes in young adults using smartphone-based sensors. Proc. ACM Interact. Mob. Wearable Ubiq. Technol. 1, 2 (June 2017). DOI : https: //doi.org/10.1145/3090051

[10] Jakob E. Bardram and Mads Frost. 2016. The personal health technology design space. IEEE Pervas. Comput. 15, 2 (2016), 70-78.

[11] Sage Bionetworks. (2018). Bridge Sensing Platform. Retrieved March 2, 2019 from https://developer.sagebridge.org/articles/overview. html.

[12] Brian M. Bot, Christine Suver, Elias Chaibub Neto, Michael Kellen, Arno Klein, Christopher Bare, Megan Doerr, Abhishek Pratap, John Wilbanks, E. Ray Dorsey, et al. 2016. The mPower study, Parkinson disease mobile data collected using ResearchKit. Sci. Data 3 (2016), 160011.

[13] Waylon Brunette, Rita Sodt, Rohit Chaudhri, Mayank Goel, Michael Falcone, Jaylen Van Orden, and Gaetano Borriello. 2012. Open data kit sensors: A sensor integration framework for Android at the application-level. In Proceedings of the 10th International Conference on Mobile Systems, Applications, and Services. ACM, 351-364.

[14] Lora E. Burke, Jun Ma, Kristen M. J. Azar, Gary G. Bennett, Eric D. Peterson, Yaguang Zheng, William Riley, Janna Stephens, Svati H. Shah, Brian Suffoletto, et al. 2015. Current science on consumer use of mobile health for cardiovascular disease prevention: A scientific statement from the American Heart Association. Circulation 132, 12 (2015), 1157-1213.

[15] Rohit Chaudhri, Waylon Brunette, Bruce Hemingway, and Gaetano Borriello. 2013. ODK sensors: An application-level sensor framework for Android devices. In Proceedings of the 3rd ACM Symposium on Computing for Development. ACM, 30.

[16] Delphine Christin, Andreas Reinhardt, Salil S. Kanhere, and Matthias Hollick. 2011. A survey on privacy in mobile participatory sensing applications. F. Syst. Softw. 84, 11 (2011), 1928-1946.

[17] Chia-Fang Chung, Jonathan Cook, Elizabeth Bales, Jasmine Zia, and Sean A. Munson. 2015. More than telemonitoring: Health provider use and nonuse of life-log data in irritable bowel syndrome and weight management. F. Med. Internet Res. 17, 8 (2015), e203.

[18] European Commission. 2019. Data protection. Retrieved March 2, 2019 from https://ec.europa.eu/info/law/law-topic/data-protection en.

[19] IEEE Standards Coordinating Committee et al. 1990. IEEE standard glossary of software engineering terminology (IEEE Std 610.121990).. IEEE Computer Society Los Alamitos, CA.

[20] Confluent. 2019. Kafka Connect / Confluent. Retrieved February 28, 2019 from https://www.confluent.io/product/connectors/.

[21] Mary Jo Deering, Erin Siminerio, and Scott Weinstein. 2013. Issue Brief: Patient-generated Health Data and Health IT. Office of the National Coordinator for Health Information Technology.

[22] David Daniel Ebert, Pim Cuijpers, Ricardo F. Muñoz, and Harald Baumeister. 2017. Prevention of mental health disorders using internetand mobile-based interventions: A narrative review and recommendations for future research. Front. Psychiat. 8 (2017), 116. 
[23] Certification Europe. 2019. BS 10012 Personal Information Management System. Retrieved March 2, 2019 from https://www. certificationeurope.com/certification/bs-10012-personal-information-management-systems/.

[24] Denzil Ferreira, Vassilis Kostakos, and Anind K. Dey. 2015. AWARE: Mobile context instrumentation framework. Front. ICT 2 (2015), 6.

[25] Fitbit. 2015. Fitbit. Retrieved March 5, 2019 from https://www.fitbit.com.

[26] Funf. 2019. Funf. Retrieved March 2, 2019 from http://www.funf.org/about.html.

[27] Andrea Gaggioli, Giovanni Pioggia, Gennaro Tartarisco, Giovanni Baldus, Daniele Corda, Pietro Cipresso, and Giuseppe Riva. 2013. A mobile data collection platform for mental health research. Pers. Ubiq. Comput. 17, 2 (Feb. 2013), 241-251. DOI: https://doi.org/10.1007/ s00779-011-0465-2

[28] GoogleFit. 2014. Google Fit. Retrieved April 2, 2019 from https://www.google.com/fit/.

[29] Mohammad Hashemian, Dylan Knowles, Jonathan Calver, Weicheng Qian, Michael C. Bullock, Scott Bell, Regan L. Mandryk, Nathaniel Osgood, and Kevin G. Stanley. 2012. iEpi: An end to end solution for collecting, conditioning and utilizing epidemiologically relevant data. In Proceedings of the 2nd ACM International Workshop on Pervasive Wireless Healthcare. ACM, 3-8.

[30] HealthKit. 2014. HealthKit. Retrieved April 2, 2019 from https://developer.apple.com/healthkit/.

[31] Netzahualcóyotl Hernández, Bert Arnrich, Jesús Favela, Cem Ersoy, Burcu Demiray, and Jesús Fontecha. 2019. A multi-site study on walkability, data sharing and privacy perception using mobile sensing data gathered from the mk-sense platform. F. Amb. Intell. Hum. Comput. 10, 6 (2019), 2199-2211.

[32] John Hicks, Nithya Ramanathan, Donnie Kim, Mohamad Monibi, Joshua Selsky, Mark Hansen, and Deborah Estrin. 2010. AndWellness: An open mobile system for activity and experience sampling. In Proceedings of the Wireless Health Conference. ACM, 34-43.

[33] Mark D. Hill. 1990. What is scalability?ACM SIGARCH Comput. Archit. News 18, 4 (1990), 18-21.

[34] Health Level 7 (HL7). 2016. HL7 Mobile Health. Retrieved December 2, 2019 from http://www.hl7.org/Special/committees/mobile/index. cfm.

[35] Syed Monowar Hossain, Timothy Hnat, Nazir Saleheen, Nusrat Jahan Nasrin, Joseph Noor, Bo-Jhang Ho, Tyson Condie, Mani Srivastava, and Santosh Kumar. 2017. mCerebrum: A mobile sensing software platform for development and validation of digital biomarkers and interventions. In Proceedings of the 15th ACM Conference on Embedded Network Sensor Systems. ACM, 7.

[36] Karen Hovsepian, Mustafa al'Absi, Emre Ertin, Thomas Kamarck, Motohiro Nakajima, and Santosh Kumar. 2015. cStress: Towards a gold standard for continuous stress assessment in the mobile environment. In Proceedings of the ACM International foint Conference on Pervasive and Ubiquitous Computing. ACM, 493-504.

[37] Cheng-Kang Hsieh, Hongsuda Tangmunarunkit, Faisal Alquaddoomi, John Jenkins, Jinha Kang, Cameron Ketcham, Brent Longstaff, Joshua Selsky, Betta Dawson, Dallas Swendeman, et al. 2013. Lifestreams: A modular sense-making toolset for identifying important patterns from everyday life. In Proceedings of the 11th ACM Conference on Embedded Networked Sensor Systems. ACM, 5.

[38] Intel. 2015. Intel Context Sensing SDK. Retrieved March 12, 2019 from https://software.intel.com/en-us/context-sensing-sdk/features.

[39] Kleomenis Katevas, Hamed Haddadi, and Laurissa Tokarchuk. 2014. Poster: Sensingkit: A multi-platform mobile sensing framework for large-scale experiments. In Proceedings of the 20th Annual International Conference on Mobile Computing and Networking. ACM, 375-378.

[40] Alexander D. Kent and Lorie M. Liebrock. 2011. Secure communication via shared knowledge and a salted hash in ad-hoc environments. In Proceedings of the IEEE 35th Annual Computer Software and Applications Conference Workshops. IEEE, 122-127.

[41] Jayden Khakurel, Helinä Melkas, and Jari Porras. 2018. Tapping into the wearable device revolution in the work environment: A systematic review. Inf. Technol. People 31, 3 (2018), 791-818.

[42] Wazir Zada Khan, Yang Xiang, Mohammed Y. Aalsalem, and Quratulain Arshad. 2012. Mobile phone sensing systems: A survey. IEEE Commun. Surv. Tutor. 15, 1 (2012), 402-427.

[43] Barbara Kitchenham and Stuart Charters. 2007. Guidelines for performing systematic literature reviews in software engineering. Technical Report EBSE-2007-01. Software Engineering Group, School of Computer Science and Mathematics, Keele University and Department of Computer Science, University of Durham.

[44] Dylan L. Knowles, Kevin G. Stanley, and Nathaniel D. Osgood. 2014. A field-validated architecture for the collection of health-relevant behavioural data. In Proceedings of the IEEE International Conference on Healthcare Informatics. IEEE, 79-88.

[45] Youngki Lee, S. S. Iyengar, Chulhong Min, Younghyun Ju, Taiwoo Park, Jinwon Lee, Yunseok Rhee, and Junehwa Song. 2012. Mobicon: A mobile context-monitoring platform. Commun. ACM 55, 3 (2012), 54.

[46] Young-Hee Lee and Ryang-Hee Kim. 2018. Estimation of the smartphone user-Satisfaction and customer intention on the social networking service. In Proceedings of the International Conference on Applied Human Factors and Ergonomics. Springer, 262-271.

[47] Xinyi Li, Elizabeth Vera, Mark Gilbert, Orieta Celiku, and Terri Armstrong. 2018. INNV-41. My STORI-a symptom tracking and reporting instrument mobile application for central nervous system cancer patients.Neuro-Oncol. 20 (11 2018), vi146-vi146. DOI : https: //doi.org/10.1093/neuonc/noy148.609

[48] Jong Hyun Lim, Andong Zhan, Evan Goldschmidt, JeongGil Ko, Marcus Chang, and Andreas Terzis. 2012. HealthOS: A platform for pervasive health applications. In Proceedings of the 2nd ACM Workshop on Mobile Systems, Applications, and Services for HealthCare. ACM, 4. 
[49] Felix Xiaozhu Lin, Ahmad Rahmati, and Lin Zhong. 2010. Dandelion: A framework for transparently programming phone-centered wireless body sensor applications for health. In Proceedings of the Wireless Health Conference (WH'10). ACM, New York, NY, 74-83. DOI : https://doi.org/10.1145/1921081.1921091

[50] Carissa A. Low, Anind K. Dey, Denzil Ferreira, Thomas Kamarck, Weijing Sun, Sangwon Bae, and Afsaneh Doryab. 2017. Estimation of symptom severity during chemotherapy from passively sensed data: Exploratory study. F. Med. Internet Res. 19, 12 (19 Dec. 2017), e420. DOI : https://doi.org/10.2196/jmir.9046

[51] Hong Lu, Jun Yang, Zhigang Liu, Nicholas D. Lane, Tanzeem Choudhury, and Andrew T. Campbell. 2010. The Jigsaw continuous sensing engine for mobile phone applications. In Proceedings of the 8th ACM Conference on Embedded Networked Sensor Systems. ACM, 71-84.

[52] Rischan Mafrur, I. Gde Dharma Nugraha, and Deokjai Choi. 2015. Modeling and discovering human behavior from smartphone sensing life-log data for identification purpose. Hum.-cent. Comput. Inf. Sci. 5, 1 (12 Oct. 2015), 31. DOI : https://doi.org/10.1186/s13673-015-00497

[53] Alex Mariakakis, Edward Wang, Shwetak Patel, and Mayank Goel. 2019. Challenges in realizing smartphone-based health sensing. IEEE Pervas. Comput. 18, 2 (2019), 76-84

[54] Wendy Berry Mendes. 2018. My BP Lab. Retrieved March 2, 2019 from http://sagebionetworks.org/research-projects/my-bp-lab/

[55] Open mHealth. 2015. Case study: Post-Traumatic Stress (PTSD). Retrieved March 2, 2019 from http://www.openmhealth.org/features/ case-studies/case-study-post-traumatic-stress-ptsd/.

[56] Open mHealth. 2015. Case study: Type 1 diabetes. Retrieved March 2, 2019 from http://www.openmhealth.org/features/case-studies/ case-study-type-1-diabetes/.

[57] Open mHealth. (2019). Open mHealth. Retrieved March 5, 2019 from http://www.openmhealth.org/.

[58] Chulhong Min, Chungkuk Yoo, Youngki Lee, and Junehwa Song. 2011. Healthopia: Towards your well-being in everyday life. In Proceedings of the 4th International Symposium on Applied Sciences in Biomedical and Communication Technologies. ACM, 108.

[59] Xiaoyun Mo, Dianxi Shi, Ruosong Yang, Han Li, ZheHang Tong, and Feng Wang. 2015. A framework of fine-grained mobile sensing data collection and behavior analysis in an energy-configurable way. In Proceedings of the IEEE International Conference on Smart City/SocialCom/SustainCom (SmartCity'15). IEEE, 391-398.

[60] W. Morrison, L. Guerdan, J. Kanugo, T. Trull, and Y. Shang. 2018. TigerAware: An innovative mobile survey and sensor data collection and analytics system. In Proceedings of the IEEE 3rd International Conference on Data Science in Cyberspace (DSC'18). 115-122. DOI : https: //doi.org/10.1109/DSC.2018.00025

[61] Institute of Electrical and Electronics Engineers. 2019. IEEE 11073 Personal health devices. Retrieved December 2, 2019 from https: //standards.ieee.org/standard/11073-00103-2012.html.

[62] Institute of Electrical and Electronics Engineers. 2019. Standard for Mobile Health Data. Retrieved December 2, 2019 from https:// standards.ieee.org/project/1752.html.

[63] National Institute of Standards and Technology. 2001. Advanced Encryption Standard (AES). Retrieved March 2, 2019 from https: //www.nist.gov/publications/advanced-encryption-standard-aes.

[64] Charith Perera, Prem Prakash Jayaraman, Arkady Zaslavsky, Peter Christen, and Dimitrios Georgakopoulos. 2014. MOSDEN: An internet of things middleware for resource constrained mobile devices. In Proceedings of the 47th Hawaii International Conference on System Sciences. IEEE, 1053-1062.

[65] Pascal B. Pfiffner, Isaac Pinyol, Marc D. Natter, and Kenneth D. Mandl. 2016. C3-PRO: Connecting ResearchKit to the health system using i2b2 and FHIR. PloS One 11, 3 (2016), e0152722.

[66] Kiran K. Rachuri, Mirco Musolesi, Cecilia Mascolo, Peter J. Rentfrow, Chris Longworth, and Andrius Aucinas. 2010. EmotionSense: A mobile phones based adaptive platform for experimental social psychology research. In Proceedings of the 12th ACM International Conference on Ubiquitous Computing. ACM, 281-290.

[67] Satish Ramkumar, Nitesh Nerlekar, Daniel D’Souza, Derek J. Pol, Jonathan M. Kalman, and Thomas H. Marwick. 2018. Atrial fibrillation detection using single lead portable electrocardiographic monitoring: A systematic review and meta-analysis. BMF Open 8, 9 (2018), e024178.

[68] Yatharth Ranjan, Maximilian Kerz, Zulqarnain Rashid, Sebastian Böttcher, Richard J. B. Dobson, and Amos A. Folarin. 2018. RADARbase: A novel open source $\mathrm{m}$-health platform. In Proceedings of the ACM International foint Conference and International Symposium on Pervasive and Ubiquitous Computing and Wearable Computers. ACM, 223-226.

[69] Reza Rawassizadeh, Martin Tomitsch, Katarzyna Wac, and A. Min Tjoa. 2013. UbiqLog: A generic mobile phone-based life-log framework. Person. Ubiq. Comput. 17, 4 (2013), 621-637.

[70] ResearchKit. 2015. ResearchKit. Retrieved March 2, 2019 from http://researchkit.org/.

[71] ResearchStack. 2016. ResearchStack. Retrieved March 5, 2019 from http://researchstack.org/.

[72] Purple Robot. 2015. Purple Robot. Retrieved March 2, 2019 from https://tech.cbits.northwestern.edu/purple-robot/.

[73] Darius A. Rohani, Nanna Tuxen, Lars V. Kessing, and Jakob E. Bardram. 2017. Designing for hourly activity sampling in behavioral activation. In Proceedings of the 11th EAI International Conference on Pervasive Computing Technologies for Healthcare (PervasiveHealth'17). ACM, New York, NY, 431-435. DOI : https://doi.org/10.1145/3154862.3154919. 
[74] Ángel Ruiz-Zafra, Kawtar Benghazi, Manuel Noguera, and José Luis Garrido. 2013. Zappa: An open mobile platform to build cloudbased m-health systems. In Ambient Intelligence-software and Applications. Springer, 87-94.

[75] Sohrab Saeb, Mi Zhang, Christopher J. Karr, Stephen M. Schueller, Marya E. Corden, Konrad P. Kording, and David C. Mohr. 2015. Mobile phone sensor correlates of depressive symptom severity in daily-life behavior: An exploratory study. 7. Med. Internet Res. 17, 7 (2015), e175.

[76] Nazir Saleheen, Amin Ahsan Ali, Syed Monowar Hossain, Hillol Sarker, Soujanya Chatterjee, Benjamin Marlin, Emre Ertin, Mustafa Al'Absi, and Santosh Kumar. 2015. puffMarker: A multi-sensor approach for pinpointing the timing of first lapse in smoking cessation. In Proceedings of the ACM International foint Conference on Pervasive and Ubiquitous Computing. ACM, 999-1010.

[77] Akio Sashima, Yutaka Inoue, Takeshi Ikeda, Tomohisa Yamashita, and Koichi Kurumatani. 2008. CONSORTS-S: A mobile sensing platform for context-aware services. In Proceedings of the International Conference on Intelligent Sensors, Sensor Networks and Information Processing. IEEE, 417-422.

[78] Akio Sashima, Yutaka Inoue, Takeshi Ikeda, Tomohisa Yamashita, Masayuki Ohta, and Koichi Kurumatani. 2008. Toward mobile healthcare services by using everyday mobile phones. In Proceedings of the 1st International Conference on Health Informatics. 242-245.

[79] M. Schickler, R. Pryss, M. Stach, J. Schobel, W. Schlee, T. Probst, B. Langguth, and M. Reichert. 2017. An IT platform enabling remote therapeutic interventions. In Proceedings of the IEEE 30th International Symposium on Computer-Based Medical Systems (CBMS'17). 111-116. DOI : https://doi.org/10.1109/CBMS.2017.78.

[80] Johannes Schobel, Rüdiger Pryss, Marc Schickler, Martina Ruf-Leuschner, Thomas Elbert, and Manfred Reichert. 2016. End-user programming of mobile services: Empowering domain experts to implement mobile data collection applications. In Proceedings of the IEEE International Conference on Mobile Services (MS'16). IEEE, 1-8.

[81] Johannes Schobel, Rüdiger Pryss, Wolfgang Wipp, Marc Schickler, and Manfred Reichert. 2016. A mobile service engine enabling complex data collection applications. In Proceedings of the International Conference on Service-oriented Computing. Springer, 626-633.

[82] Audacious Software. 2016. Passive Data Kit. Retrieved March 2, 2019 from https://audacious-software.com/passive-data-kit/.

[83] Callum L. Stewart, Zulqarnain Rashid, Yatharth Ranjan, Shaoxiong Sun, Richard J. B. Dobson, and Amos A. Folarin. 2018. RADARbase: Major depressive disorder and epilepsy case studies. In Proceedings of the ACM International foint Conference and International Symposium on Pervasive and Ubiquitous Computing and Wearable Computers. ACM, 1735-1743.

[84] Hongsuda Tangmunarunkit, Cheng-Kang Hsieh, Brent Longstaff, S. Nolen, John Jenkins, Cameron Ketcham, Joshua Selsky, Faisal Alquaddoomi, Dony George, Jinha Kang, et al. 2015. Ohmage: A general and extensible end-to-end participatory sensing platform. ACM Trans. Intell. Syst. Technol. 6, 3 (2015), 38.

[85] John Torous, Mathew V. Kiang, Jeanette Lorme, and Jukka-Pekka Onnela. 2016. New tools for new research in psychiatry: A scalable and customizable platform to empower data driven smartphone research. FMIR Ment. Health 3, 2 (2016).

[86] Jiangtao Wang, Yasha Wang, Daqing Zhang, and Sumi Helal. 2018. Energy saving techniques in mobile crowd sensing: Current state and future opportunities. IEEE Commun. Mag. 56, 5 (2018), 164-169.

[87] Rui Wang, Fanglin Chen, Zhenyu Chen, Tianxing Li, Gabriella Harari, Stefanie Tignor, Xia Zhou, Dror Ben-Zeev, and Andrew T. Campbell. 2014. StudentLife: Assessing mental health, academic performance and behavioral trends of college students using smartphones. In Proceedings of the ACM International foint Conference on Pervasive and Ubiquitous Computing. ACM, 3-14.

[88] Dan E. Webster, Christine Suver, Megan Doerr, Erin Mounts, Lisa Domenico, Tracy Petrie, Sancy A. Leachman, Andrew D. Trister, and Brian M. Bot. 2017. The mole mapper study, mobile phone skin imaging and melanoma risk data collected using ResearchKit. Sci. Data 4 (2017), 170005.

[89] Peter West, Max Van Kleek, Richard Giordano, Mark J. Weal, and Nigel Shadbolt. 2018. Common barriers to the use of patient-generated data across clinical settings. In Proceedings of the CHI Conference on Human Factors in Computing Systems. ACM, 484.

[90] Claes Wohlin. 2014. Guidelines for snowballing in systematic literature studies and a replication in software engineering. In Proceedings of the 18th International Conference on Evaluation and Assessment in Software Engineering. ACM, 38.

[91] Pang Wu, Huan-Kai Peng, Jiang Zhu, and Ying Zhang. 2011. Senscare: Semi-automatic activity summarization system for elderly care. In Proceedings of the International Conference on Mobile Computing, Applications, and Services. Springer, 1-19.

[92] Pang Wu, Jiang Zhu, and Joy Ying Zhang. 2013. MobiSens: A versatile mobile sensing platform for real-world applications. Mob. Netw. Applic. 18, 1 (2013), 60-80.

[93] X. Xia, D. Lo, F. Zhu, X. Wang, and B. Zhou. 2013. Software internationalization and localization: An industrial experience. In Proceedings of the 18th International Conference on Engineering of Complex Computer Systems. 222-231. DOI : https://doi.org/10.1109/ICECCS.2013.40.

[94] Haoyi Xiong, Yu Huang, Laura E. Barnes, and Matthew S. Gerber. 2016. Sensus: A cross-platform, general-purpose system for mobile crowdsensing in human-subject studies. In Proceedings of the ACM International foint Conference on Pervasive and Ubiquitous Computing. ACM, 415-426.

Received December 2019; revised May 2020; accepted July 2020 